\title{
Aircraft Control-Display Analysis and Design Using the Optimal Control Model of the Human Pilot
}

\author{
RONALD A. HESS
}

\begin{abstract}
The use of the optimal control model (OCM) of the human pilot as a design tool is discussed. A novel procedure for the assignment and selection of model parameters in the absence of experimental data is proposed. A technique for partitioning a weakly coupled, multiaxis task into approximate state-uncoupled, single-axis tasks is introduced. The resulting modeling technique is utilized in the design and analysis of an aircraft flight-director system. This flight-director design technique differs from previous related work using the OCM in that considerable effort is devoted to ensuring that the OCM-designed director exhibits the desirable frequency-domain characteristics associated with experimentally verified classical designs (e.g., $K / s$ "effective vehicle" characteristics, noninteracting controls, etc.). The implications of the technique in the design of automatic flight control systems which employ the human pilot as a performance assessor and failure detector are briefly discussed.
\end{abstract}

\section{INTRODUCTION}

$\mathrm{T}$ HE PAST decade has witnessed the increasing utilization of the optimal control model (OCM) of the human operator in the analysis of human performance and behavior in man-machine systems [1]-[3]. The use of the OCM as a design tool has progressed also, albeit more slowly [4]-[7]. This is attributable to the difficult problem of selecting OCM index of performance weighting coefficients and noise covariances in the absence of corroborative experimental data, especially in the case of multioutput systems.

In this paper consideration is given to the utility of the OCM as a control-display design tool with particular emphasis on its use in the design of aircraft flight directors. We will first simplify the model, and then, focusing attention on single-axis (control) tasks, we will discuss an "equivalent time-constant" method to relate the "maximum allowable deviations" of pilot control rates to the rates of change of vehicle outputs. Hypothetical performance-workload trade-offs will be employed to uniquely specify the desired equivalent time constants and observation noises (or task attention parameters). Finally, an open-loop eigenanalysis technique will allow us to partition weakly coupled multiaxis tasks into constituent single-axis tasks for the purpose of selecting index of performance weighting coefficients.

Manuscript received September 9, 1980; revised April 17, 1981. This paper was presented at the 1980 International Conference on Cybernetics and Society.

The author is with the Aircraft Guidance and Navigation Branch, Ames Research Center, National Aeronautics and Space Administration, Moffett Field, CA 94035

\section{Pilot Modeling: Single-Axis (Control), Single-OUTPUT TASKS}

Before beginning a discussion of the modeling technique, a very brief review of the OCM is in order. A detailed discussion of the model is beyond the scope of this paper since a wealth of pertinent information exists in the literature, both in terms of theory and application [1]-[3]. Fig. 1 is a general block diagram representation of the OCM. For the purposes of the analysis technique to be described, the OCM will be completely specified when the following parameters have been selected: 1) the time delay $\tau$; 2) the noise-to-signal ratio for each control, $\rho_{j}^{\prime}$; 3) the noise-tosignal ratio for each observed variable, $\rho_{i}$; and 4) the weighting coefficients in the index of performance.

For the sake of simplicity, we will set $\tau=0.2 \mathrm{~s}$ and $\rho_{j}^{\prime}=0.0001(-40 \mathrm{~dB})$ and will not allow them to vary in the analyses to follow. An expression for the noise-to-signal ratios for the observed variables will be given as

$$
\rho_{i}=\frac{0.01}{a_{c} / 2 d_{n}}, \quad i=1,2, \cdots,\left(2 d_{n}\right)
$$

where

$\rho_{i} \quad$ noise-to-signal ratio for the $i$ th observed variable.

$a_{c}$ attention parameter, whose value is assumed to be proportional to the amount of attention the pilot is allocating to the control task.

$d_{n}$ number of explicitly displayed variables.

In keeping with the established structure of the OCM, we assume the pilot can perceive the first derivative of an explicitly displayed variable but not higher derivatives. The collection of explicitly displayed variables and their firsttime derivatives will be referred to as observed variables. Although analytical methods for including the effect of visual indifference thresholds and nonuniform attention allocation exist [3], for expediency none will be utilized in the technique to be described. The appearance of the factor $1 / 2 d_{n}$ in the denominator of (1) stems from the assumption that the entire control task attention $\left(a_{c}\right)$ is distributed evenly over all the observed variables $\left(2 d_{n}\right.$ in number). Thus, selection of $a_{c}$ is equivalent to selection of $\rho_{i}$, and the former will be a fundamental parameter in our analyses.

Values of the weighting coefficients of the index of performance will be based on reciprocals of "maximum allowable deviations" of the respective variables [8] chosen 


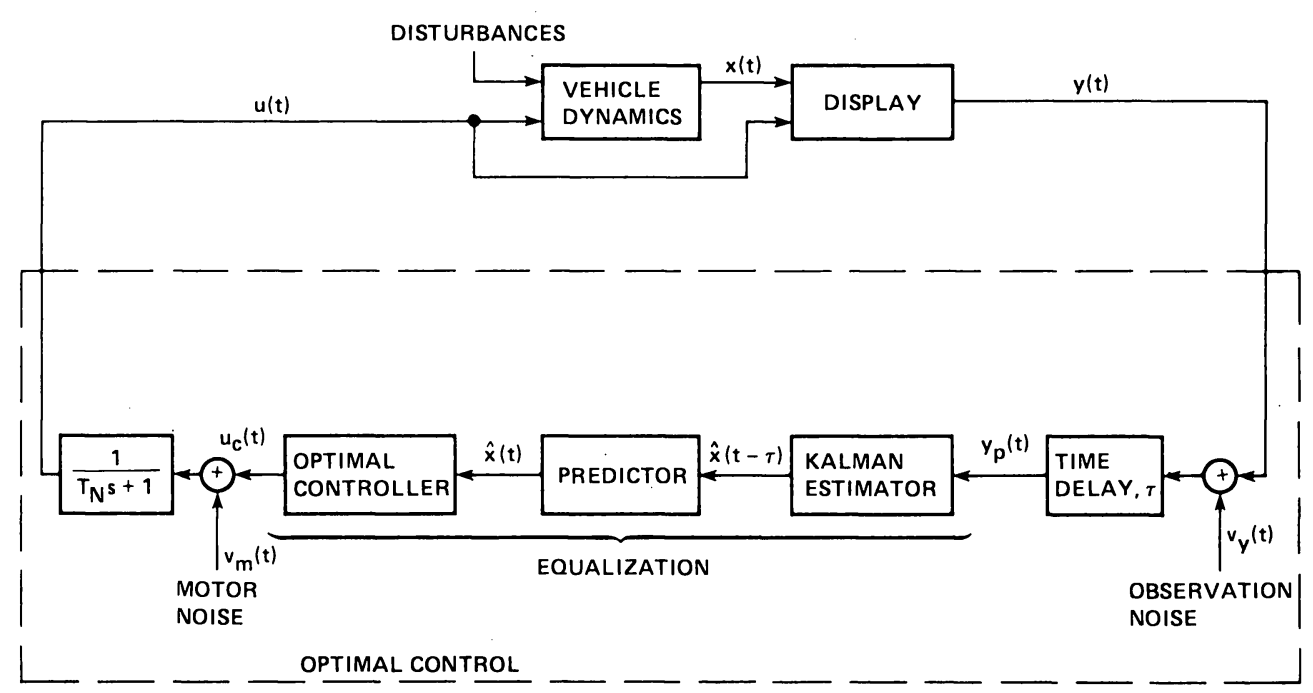

Fig. 1. Optimal control model (OCM) of the human pilot.

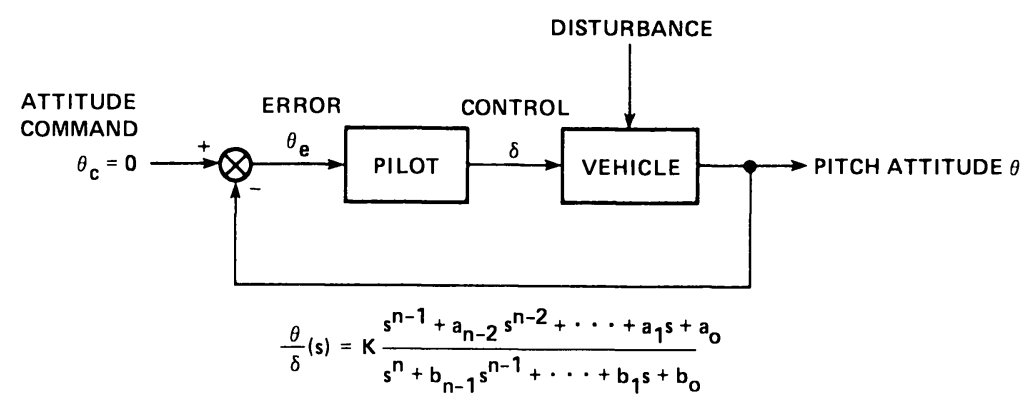

Fig. 2. Single-axis (control), single-variable task.

by what will be termed an "effective time-constant" method. Single-axis tasks will be considered first. Fig. 2 is a block diagram representation of a tracking task in which the pilot is attempting to control the pitch attitude $\theta(t)$ of some aircraft in the presence of atmospheric turbulence. This diagram will serve as a useful paradigm for all the singleaxis tasks to be discussed and for the inner loop of the multivariable tasks. For this task, the OCM index of performance will be selected as

$$
J=E\left\{\frac{\lim }{\chi \rightarrow \infty} \frac{1}{\chi} \int_{0}^{\chi}\left[\theta^{2}(t) / \theta_{M}^{2}+\dot{\delta}^{2}(t) / \dot{\delta}_{M}^{2}\right] d t\right\}
$$

The transfer function representing the system dynamics has been shown as a ratio of polynomials. Pure-time delays that might be included in a vehicle description to account for the phase effects of high-frequency dynamics or to model delays inherent in the implementation of digital control laws can be incorporated through appropriate Pade approximations. We will assign an arbitrary maximum allowable deviation to the time rate of change of the error, $\dot{\theta}(t)$, and denote it $\dot{\theta}_{M}$. Now an effective time constant $T$ can be introduced to define maximum allowable deviations of the integral and derivatives of $\dot{\theta}(t)$ and $\dot{\delta}(t)$ as

$$
\begin{aligned}
& \theta_{M}=\dot{\theta}_{M} T ; \quad \delta_{M}=\dot{\delta}_{M} T ; \\
& \begin{array}{cc}
\dot{\theta}_{M}=\underset{\text { specified but }}{\text { arbitrary; }} & \dot{\delta}_{M}=\text { to be } \\
\text { selected; }
\end{array} \\
& \ddot{\theta}_{M}=\dot{\theta}_{M} / T ; \quad \ddot{\delta}_{M}=\dot{\delta}_{M} / T ; \quad \text {. } \\
& \ddot{\theta}_{M}=\ddot{\theta}_{M} / T=\dot{\theta}_{M} / T^{2} \cdot \ddot{\delta}_{M}=\ddot{\delta}_{M} / T=\dot{\delta}_{M} / T^{2} ;
\end{aligned}
$$

Relating the maximum allowable deviations of vehicle output and pilot control variables to reciprocal powers of $T$ is justified by the pilot/vehicle bandwidth considerations which will be discussed.

Unlike $\dot{\theta}_{M}$, the value of $\dot{\delta}_{M}$ in (3) will not be arbitrary but will be found using (3) and the vehicle dynamics of Fig. 2 as follows: with zero initial conditions, the relationship between the power of the Laplace variable $s$ and the order of a time derivative is given by

$$
s^{n} \rightarrow \frac{d^{n}}{d t^{n}}
$$




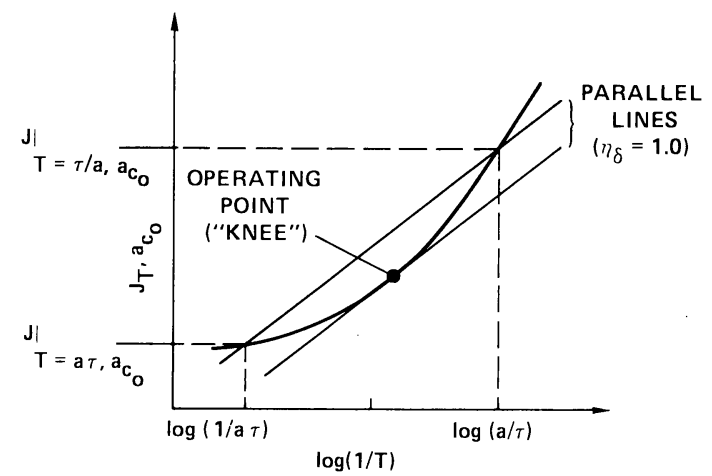

Fig. 3. Generic curve for quadratic cost versus inverse time constant (single variable case).

Using (3) and relation (4) and the algebraic expression in $s$ shown in Fig. 2, we propose the following:

$\dot{\delta}_{M}=\frac{\left[1 / T^{n-1}+\left|b_{n-1}\right| / T^{n-2}+\cdots+\left|b_{1}\right|+\left|b_{0}\right| T\right]}{K\left[1 / T^{n-2}+\left|a_{n-2}\right| / T^{n-3}+\cdots+\left|a_{1}\right|+\left|a_{0}\right| T\right]} \dot{\theta}_{M}$.

We do not mean to imply here that the integrals and derivatives of $\dot{\theta}$ and $\dot{\delta}$ actually achieve their maximum values simultaneously. Rather we allow the numerator and denominator of (5) to contain a weighted sum of the maximum values of the dependent and control input variables, respectively. As (5) indicates, the weighting factors are governed by the system dynamics. The right side of (5) can be interpreted as the maximum value of $\dot{\delta}(t)$ that the pilot is willing to generate in a tracking task, given an internal model of the system dynamics and a desired maximum value of pitch rate, $\dot{\theta}(t)$. Thus, given the vehicle dynamics and values for $\dot{\theta}_{M}$ and $T$, the analyst can find $\dot{\delta}_{M}$. This, in turn, will allow definition of the weighting coefficients in the index of performance, now denoted $J_{T, a_{c}}$.

An inspection of (2) and (5) suggests that $T$ essentially determines the bandwidth of the pilot-vehicle system. For physically realizable systems, $\dot{\delta}_{M} / \theta_{M}$ will be approximately proportional to powers of $(1 / T)$. Thus, as $(1 / T)$ is allowed to increase (decrease), $\dot{\delta}_{M} / \theta_{M}$ increases (decreases) and the quadratic performance index allows more (less) control activity in minimizing tracking error. Furthermore, with $a_{c}$ fixed at some nominal value $a_{c_{0}}$, changes in $J_{T, a_{c_{0}}}$ brought about by changes in $T$ reflect variations in quadratic performance relative to variations in the maximum allowable deviations appearing in (2). Following Hess [9], we will assume that these variations can be used to quantify the relative acceptability of the task demands from the pilot's point of view. On the other hand, with $T$ fixed at some nominal value $T_{0}$, changes in $J_{T_{0}, a_{c}}$ brought about by changes in $a_{c}$, reflect only variations in quadratic performance.

We now assert that with $a_{c}$ fixed at some $a_{c_{0}}$, selection of $T$ by the analyst should parallel a hypothetical "performance-workload" trade-off made by the pilot between his desire to maximize closed-loop disturbance suppression or command following capabilities (maximize $1 / T$ ) and his

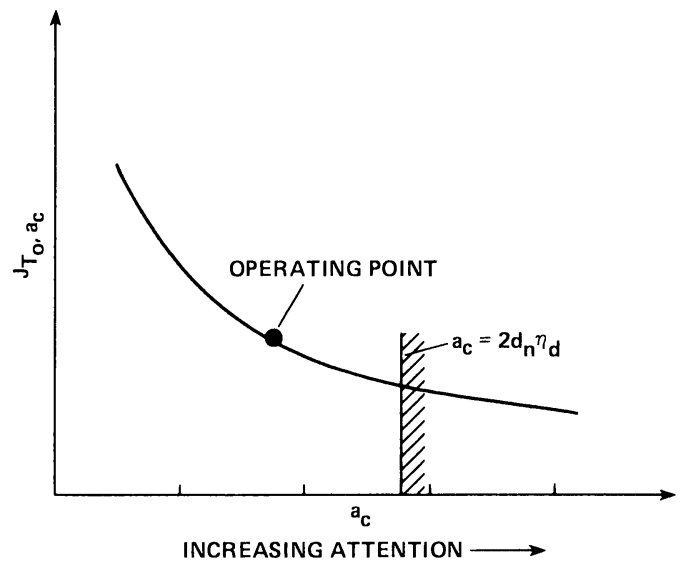

Fig. 4. Generic curve for quadratic cost versus attention parameter.

desire to maximize task acceptability (minimize $J_{T, a_{c}}$ ). As will be shown, these criteria cannot be simultaneously met; that is, a trade-off must be undertaken. Likewise, with $T$ fixed at some $T_{0}$, selection of $a_{c}$ should parallel a hypothetical "performance-workload" trade-off between the pilot's desire to maximize closed-loop system performance (minimize $\left.J_{T_{0}, a_{\mathrm{c}}}\right)$ and his desire to minimize "attentional demand" (minimize $a_{c}$ ).

In [10], a simple technique was introduced to allow the analyst to accomplish the hypothetical trade-off which it is assumed the pilot makes by first selecting a domain of $1 / T$ containing the reciprocal of the pilot's time delay, $\tau$ (i.e., $(1 / a \tau)<1 / T<(a / \tau)$, where $a>1.0)$. The operating point is then defined as the "knee" of the $J_{T, a_{c_{0}}}$ versus $\log (1 / T)$ curve, i.e., the value of $T$ where

$$
\frac{\partial J_{T, a_{c_{0}}}}{\partial \log (1 / T)}=\eta_{\delta} \frac{\left(J_{\tau / a, a_{c_{0}}}-J_{a \tau, a_{c_{0}}}\right)}{\log (a / \tau)-\log (1 / a \tau)} .
$$

Here, $\eta_{\delta}$ is a constant, nominally unity, which can be used to reflect manipulator characteristics, much like an efficiency factor. This procedure defines the operating point indicated in Fig. 3. It is important to emphasize that the ordinate in Fig. 3 does not reflect absolute performance. Each of the quadratic terms in $J_{T, a_{c_{0}}}$ are normalized by factors that are functions of $1 / T$. For example, although the magnitude of $J_{T, a_{c 0}}$ is decreasing as $1 / T$ becomes smaller for the dynamics of Fig. 3, absolute performance is actually deteriorating. With the value of $T$ corresponding to an operating point $\left(T=T_{0}\right), J_{T_{0}, a_{c}}$ versus $a_{c}$ may be drawn as shown generically in Fig. 4. Again, a procedure for allowing the analyst to accomplish the hypothetical trade-off made by the pilot is to select a domain of $a_{c}$, $a_{c_{1}}<a_{c}<a_{c_{2}}$, where

$$
\begin{aligned}
a_{c_{1}}= & \text { value of } a_{c} \text { for which } \\
& {\left[1 / J_{T_{0}, a_{c}}\right]\left[\partial J_{T_{0}, a_{c}} / \partial a_{c}\right]>\alpha } \\
a_{c_{2}}= & \text { value of } a_{c} \text { for which } \\
& {\left[1 / J_{T_{0}, a_{c}}\right]\left[\partial J_{T_{0}, a_{c}} / \partial a_{c}\right]<\beta }
\end{aligned}
$$

and where $\alpha$ and $\beta$ are constants, and $\alpha<\beta$. The operating point is then defined as the knee of the curve $J_{T_{0}, a_{c}}$ 


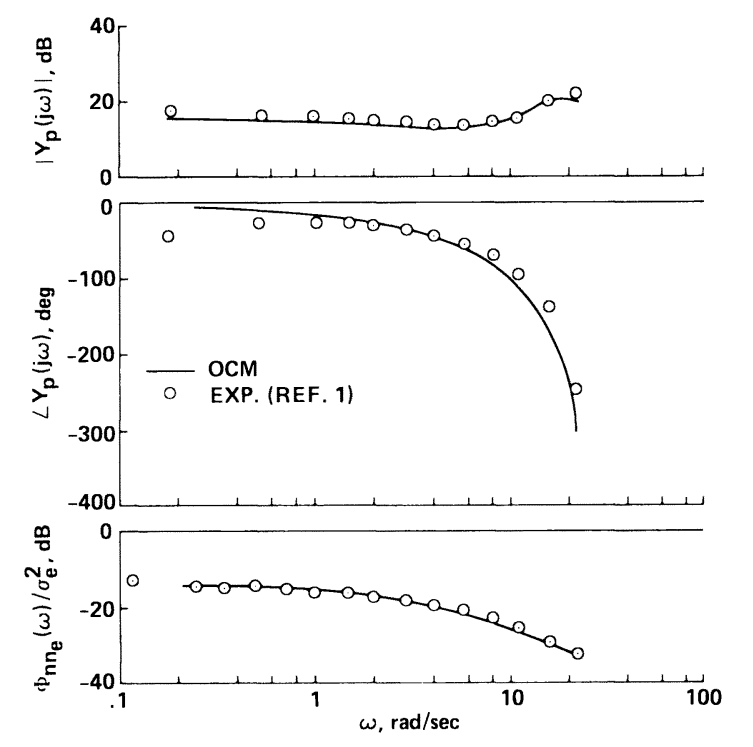

Fig. 5. OCM and experimental frequency domain measures, $K / s$ dynamics (average of four subjects).

versus $a_{c}$, i.e., the value of $a_{c}$ where

$$
\frac{\partial J_{T_{0}, a_{c}}}{\partial a_{c}}=\frac{\left(J_{T_{0}, a_{c_{2}}}-J_{T_{0}, a_{c_{1}}}\right)}{a_{c_{2}}-a_{c_{1}}} .
$$

To eliminate the possibility of unrealistically small observation noise-to-signal ratios from being selected with this technique, we shall also require that

$$
a_{c}<2\left(d_{n}\right) \eta_{d}
$$

Here, $\eta_{d}$ is a constant, nominally unity, which can be used to reflect general display characteristics, again, much like an efficiency factor. The value of $a_{c}$ corresponding to the operating point defined by (7)-(9) is shown in Fig. 4. If this value is different from the value $a_{c_{0}}$ used in selecting $T_{0}$, then the curves of Fig. 3 can be generated again with the new value of $a_{c}$. Note that this technique for selecting operating points is completely independent of the value assigned to the maximum allowable deviation on error rate $\dot{\theta}_{M}$. Of course this does not imply that this variable is unimportant. Rather, there exists an inherent scaling effect in the technique that renders it insensitive to the particular value of $\dot{\theta}_{M}$ chosen by the analyst. In all the tasks of [10] and those to be discussed here, $a=4, \alpha=0.1$, and $\beta=0.5$.

Fig. 5 shows the OCM transfer function and remnant power spectral density which was generated by the technique just described compared with experimental values taken from [1]. The OCM results also compare very well with those of [1]. The controlled element dynamics were $K / s$. The analysis technique and experimental comparisons are discussed in further detail in [10]. These results indicate that for many engineering applications, this OCM technique yields acceptable models of the human pilot in single-axis, single-variable tracking tasks.

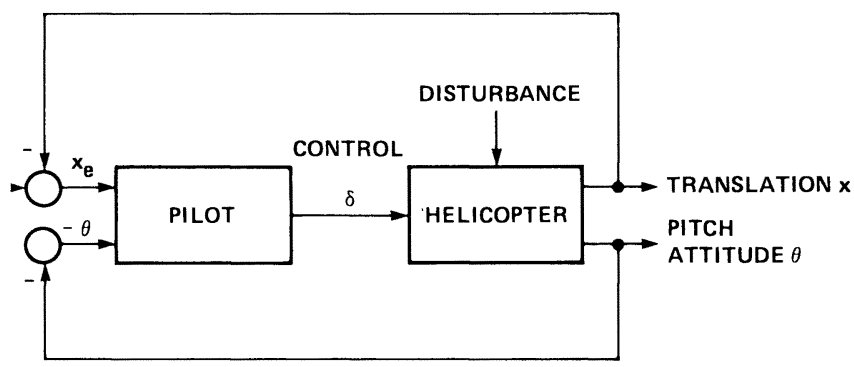

Fig. 6. Single-axis (control), multivariable task.

\section{Pilot Modeling: Single-Axis (Control), MultioutPUT TASKS}

Fig. 6 is a block diagram of a helicopter longitudinal precision hover task (single-axis, multivariable task). The obvious difference between this and the task of Fig. 2 is the multivariable nature of the hover task; that is, two vehicle output variables are controlled by the pilot: pitch attitude $\theta(t)$ and longitudinal translation $x(t)$. The OCM technique utilized in the previous examples must obviously be extended now to multivariable tasks. This can be accomplished in the context of effective time constants by considering the system of Fig. 6 as a rudimentary example of a two-level hierarchical control structure. The outer level or loop consists of vehicle translation and the inner level or loop consists of vehicle attitude. We will assign an effective time constant to each of these levels and denote then $T_{\text {out }}$ and $T_{\text {in }}$. The outer-loop and inner-loop dichotomy naturally suggests $T_{\text {out }}>T_{\text {in }}$.

For the hovering helicopter, we can write

$$
\frac{x}{\theta}(s)=\frac{X_{u}\left(s^{2}-M_{q} s\right)-M_{u} g}{M_{u} s^{2}},
$$

where $X_{u}, M_{u}$, and $M_{q}$ represent normalized vehicle aerodynamic stability derivatives [11]. Equation (10) yields the differential equation

$$
\ddot{x}=\left(X_{u} / M_{u}\right) \ddot{\theta}-\left(M_{q} / M_{u}\right) X_{u} \dot{\theta}-g \theta
$$

Proceeding as in (3)-(5), we write the algebraic relation

$$
\ddot{x}_{M}=\left|X_{u} / M_{u}\right| \dot{\theta}_{M} / T_{\text {in }}+\left|M_{q} X_{u} / M_{u}\right| \dot{\theta}_{M}+|g| \dot{\theta}_{M} T_{\text {in }} .
$$

Here, with $T_{\text {in }}$ specified, $\ddot{x}_{M}$ can be thought of as the maximum allowable deviation in longitudinal acceleration that the pilot will allow, given an internal model of the vehicle dynamics and a maximum allowable deviation of pitch rate, $\dot{\theta}_{M}$. Now, $T_{\text {out }}$ can be introduced by specifying

$$
\begin{aligned}
& \dot{x}_{M}=\ddot{x}_{M} T_{\text {out }} \\
& x_{M}=\dot{x}_{M} T_{\text {out }}=\ddot{x}_{M}\left(T_{\text {out }}\right)^{2} .
\end{aligned}
$$

As defined in [10], the index of performance for the OCM 


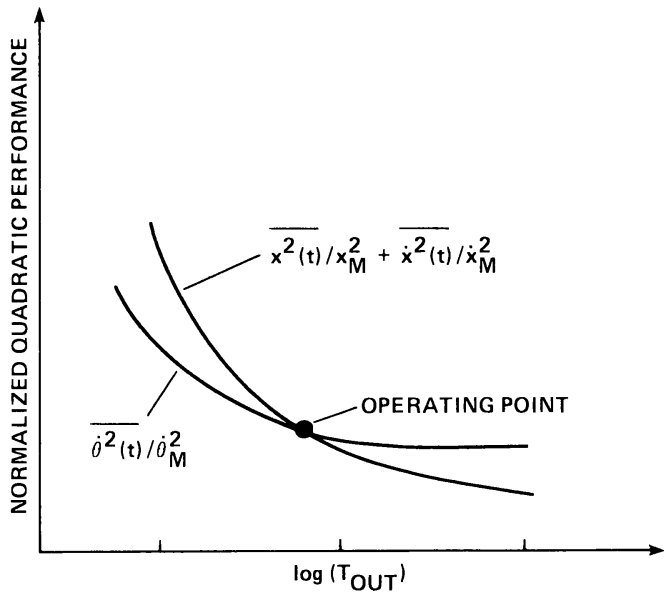

Fig. 7. Generic curves for inner- and outer-loop normalized quadratic performance versus outer-loop time constant.

procedure was selected as

$$
\begin{array}{r}
J_{T_{\text {in }}, T_{\text {out }}, a_{c}}=E\left\{\frac{\lim }{\chi \rightarrow}+\infty \frac{1}{\chi} \int_{0}^{\chi}\left[\dot{\theta}^{2}(t) / \dot{\theta}_{M}^{2}+x^{2}(t) / x_{M}^{2}\right.\right. \\
\left.\left.+\dot{x}^{2}(t) / \dot{x}_{M}+\dot{\delta}^{2}(t) / \dot{\delta}_{M}^{2}\right] d t\right\} .
\end{array}
$$

Selection of variables to appear in (13) was not arbitrary but based on the hierarchy just described and on the task definition. First, the hierarchy suggests that pitch attitude $\theta(t)$ is the primary means for controlling longitudinal translation; therefore, minimizing $\overline{\theta^{2}(t)}$, per se, may not be a reasonable control strategy. Minimizing $\overline{\dot{\theta}^{2}(t)}$, however, is important from the standpoint of pilot-passenger comfort and safety. Second, since the task was described to the simulation pilots as a "precision hover," both translation and velocity were included in the definition of the index of performance.

The maximum allowable deviations were chosen as follows. First, the hierarchy just mentioned suggests that $\dot{\delta}_{M}$ should be based on inner-loop characteristics; thus, a relation analogous to (5) was developed based on the vehicle pitch attitude dynamics. Again, an arbitrary value of $\dot{\theta}_{M}$ was chosen. Using initial estimates for $T_{\text {in }}$ and $a_{c}$, a value of $\dot{\delta}_{M}$ was calculated. Curves of normalized quadratic performance involving pitch rate and the sum of translation and velocity were drawn versus $\log \left(T_{\text {out }}\right)$, as shown in Fig. 7. The intersection of these curves provides a reasonable choice for an operating point ( $T_{\text {out }}$ value), that is, the point at which the inner-loop and outer-loop normalized quadratic performance measures are making equal contributions to the index of performance. The rationale behind this choice can be made more evident by considering the variations in absolute inner-loop and outer-loop performance that accompany variations in the magnitude of $T_{\text {out }}$. When $T_{\text {out }}$ is selected so that normalized inner-loop performance dominates the value of the index of performance ( $T_{\text {out }}$ large), absolute outer-loop performance is very poor; that is, large translational displacements and velocities occur. This is not surprising since, as $T_{\text {out }} \rightarrow \infty$, we have essentially changed the task into one of pitch rate tracking. On the other hand, when $T_{\text {out }}$ is selected so that normalized outer-loop performance dominates the value of the index of performance ( $T_{\text {out }}$ small), absolute inner-loop performance is very poor; that is, unrealistically large pitch rates are generated. In the light of this, selecting $T_{\text {out }}$ so that normalized inner-loop and outer-loop performance each contribute equally to the value of the index of performance is certainly reasonable. With the value of $T_{\text {out }}$ so chosen, we proceed to the determination of $T_{\text {in }}$ and $a_{c}$.

As (11) and (12) clearly show, $T_{\text {in }}$ affects both inner-loop and outer-loop performance through its influence on $x_{M}$ and $\dot{x}_{M}$. However, we still wish to select $T_{\text {in }}$ based on inner-loop performance variations alone. We can do this by creating an auxiliary performance metric

$$
P=\overline{\theta^{2}(t) / \theta_{M}^{2}}+\overline{\dot{\delta}^{2}(t) / \dot{\delta}_{M}^{2}}
$$

and plotting its value versus $\log \left(1 / T_{\text {in }}\right)$ in a manner completely analogous to that shown in Fig. 3. Note that $P$ is not the actual index of performance governing the OCM; it is a metric that allows us to select $T_{\text {in }}$, based on inner-loop performance when the inner and outer loops are closed in the OCM via (13). Depending on the quality of the initial estimate for $T_{\text {in }}$ and $a_{c}$, some iteration on $T_{\text {out }}$ may be necessary. Finally, the attention parameter $a_{c}$ is selected in a manner identical to that used in the single-variable tasks. As was the case with the previous tasks, the arbitrary value of $\dot{\theta}_{M}$ has no effect on the procedure used to determine $T_{\mathrm{in}}$, $T_{\text {out }}$, and $a_{c}$.

The technique for selecting index-of-performance weighting coefficients outlined here differs from that often employed in OCM design work, in which the maximum allowable deviations are chosen on the basis of task requirements. Such requirements are often obtained from pilot questionnaires, as in [12]. If this avenue is not available, the designer must simply rely upon intuition and past experience. In such cases, the method proposed here offers what the author believes to be a preferable alternative.

Fig. 8 shows a comparison between the OCM performance predictions using the model parameter selection technique just described and those from the experiments of [13]. These experiments did not include pilot describing function measurements. However, a necessary condition for the validity of any model of the human operator is that the "circulatory" or effective pilot-vehicle-display transfer function exhibit $(K / s) e^{-\tau_{e} s}$ characteristics in the region of crossover [14]. Fig. 9 shows the circulatory transfer function obtained by opening the loop of Fig. 6 at $\delta$ and calculating the resulting loop transfer function. The $(K / s) e^{-\tau_{e} s}$ characteristics of the circulatory transfer function of Fig. 9 and the excellent performance comparisons of Fig. 8 support the validity of the OCM application.

\section{Pilot Modeling: Multiaxis (Control), MultioutPUT TASKS}

Multiaxis (control), multioutput tasks are those for which the OCM seems to have the greatest potential. In this 

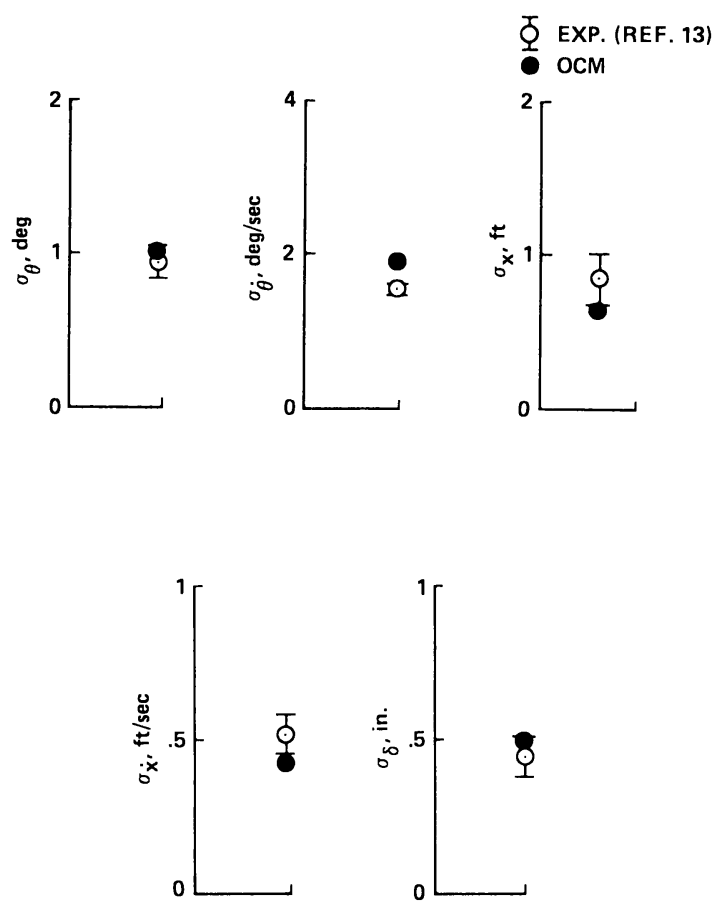

Fig. 8. Model and experimental performance comparison for helicopter hover tasks of Fig. 6.
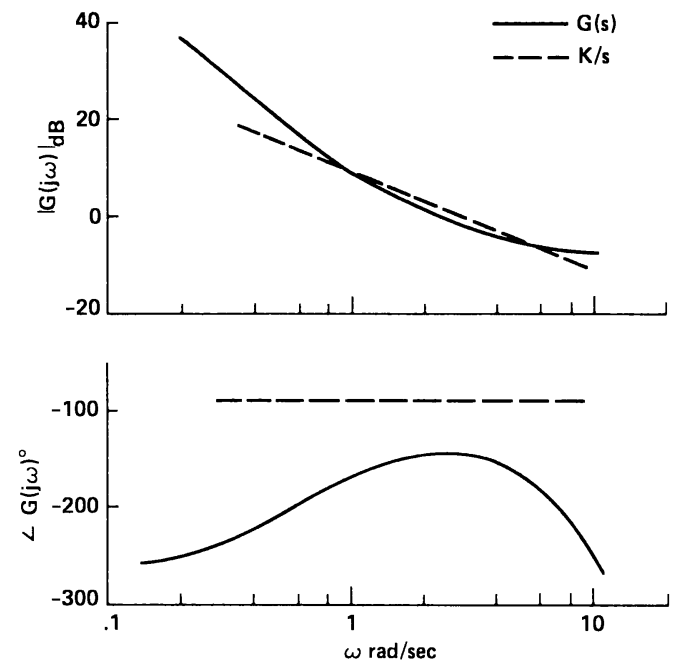

Fig. 9. Circulatory-transfer function for helicopter hover task of Fig. 6.

section we will introduce a technique for partitioning a weakly coupled, multiaxis task into approximate stateuncoupled, single-axis tasks which can be analyzed using the techniques described in the preceding sections. We will use the uncoupled approximations for the design of flight directors and to yield index of performance weighting coefficients for the analysis of the coupled system.

To discuss the partitioning scheme, let us consider the two-axis (control) manual control task shown in Fig. 10. Here, the human operator is to control the vertical and rotational motion of a large beam through two ideal actuation devices, which instantaneously apply forces $f_{1}$ and $f_{2}$ to the beam as shown in Fig. 10. The task consists of main-
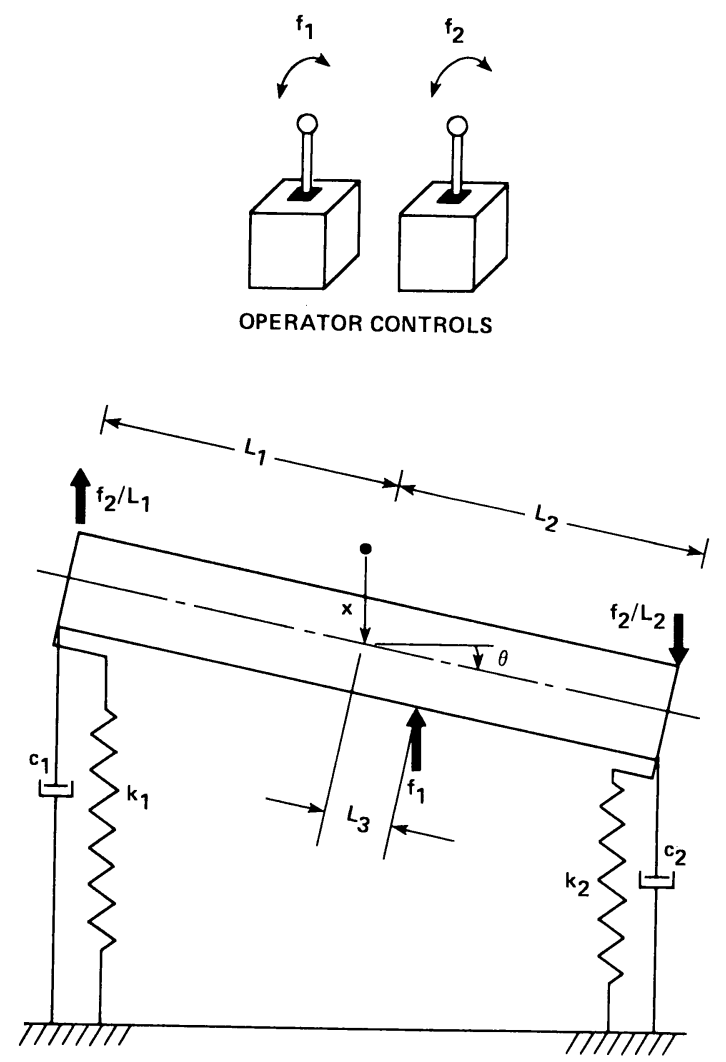

Fig. 10. Two-axis (control) manual control task.

taining the beam in an equilibrium position in the presence of random disturbing forces and moments $\delta_{f}$ and $\delta_{m}$. This rather unorthodox manual control example was chosen to illustrate the eigenanalysis to follow. The equations of motion for the system can be written

$$
\begin{aligned}
m \ddot{x}= & -\left(k_{1}+k_{2}\right) x-\left(c_{1}+c_{2}\right) \dot{x}+\left(k_{1} L_{1}-k_{2} L_{2}\right) \theta \\
& +\left(c_{1} L_{1}-c_{2} L_{2}\right) \dot{\theta}-f_{1}+\left(1 / L_{2}-1 / L_{1}\right) f_{2}+\delta_{f} \\
J \ddot{\theta}= & \left(k_{1} L_{1}-k_{2} L_{2}\right) x+\left(c_{1} L_{1}-c_{2} L_{2}\right) \dot{x} \\
& -\left(k_{1} L_{1}^{2}+k_{2} L_{2}^{2}\right) \theta-\left(c_{1} L_{1}^{2}+c_{2} L_{2}^{2}\right) \dot{\theta} \\
& +2 f_{2}-L_{3} f_{1}+\delta_{m} .
\end{aligned}
$$

To allow the human operator inputs $f_{1}$ and $f_{2}$ to appear in the eigenanalysis, we will augment (15) with

$$
\begin{aligned}
& \dot{f}_{1}=-\frac{1}{T_{N}} f_{1}+u_{1} \\
& \dot{f}_{2}=-\frac{1}{T_{N}} f_{2}+u_{2} .
\end{aligned}
$$

Here, $T_{N}$ is chosen so the bandwidths of the control "outputs" $f_{i}$ are consistent with those of the human operator, typically $T_{N}=0.1 \mathrm{~s}$. In this example, $k_{1}=k_{2}=3000$ $\mathrm{lbf} / \mathrm{ft}, c_{1}=c_{2}=150 \mathrm{lbf}-\mathrm{s} / \mathrm{ft}, L_{1}=5 \mathrm{ft}, L_{2}=7 \mathrm{ft}, L_{3}=1$ $\mathrm{ft}, m=100 \mathrm{slugs}$, and $J=2500 \mathrm{lbf} / \mathrm{s}^{2}$. Equations (15) and (16) can be written in state space format by defining 


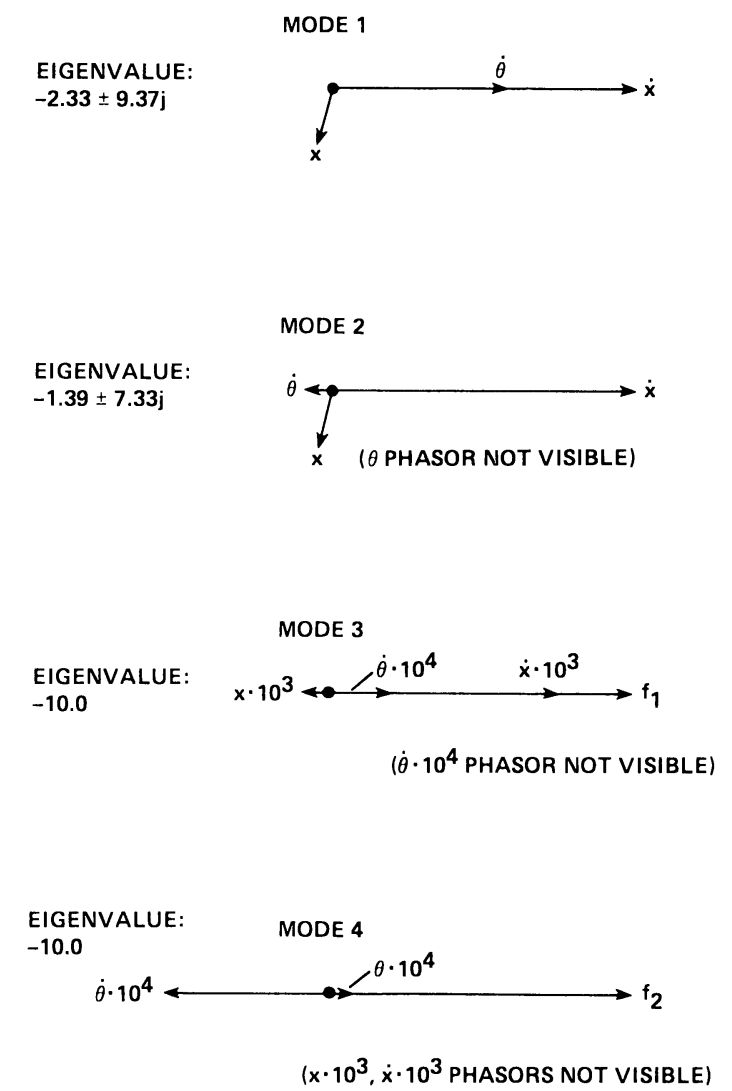

Fig. 11. Eigenvectors for system of Fig. 10.

$$
\begin{aligned}
x_{1}=f_{1}, x_{2}= & f_{2}, x_{3}=x, x_{4}=\dot{x}_{3}, x_{5}=\theta, x_{6}=\dot{x}_{5} . \text { Thus, } \\
\dot{x}_{1}= & -10 x_{1}+u_{1} \\
\dot{x}_{2}= & 10 x_{2}+u_{2} \\
\dot{x}_{3}= & x_{4} \\
\dot{x}_{4}= & -0.01 x_{1}-0.00057 x_{2}-60 x_{3}-3 x_{4} \\
& -60 x_{5}-3 x_{6}+\delta_{f} \\
\dot{x}_{5}= & x_{6} \\
\dot{x}_{6}= & -0.0004 x_{1}+0.0008 x_{2}-2.4 x_{3} \\
& -0.12 x_{4}-88.8 x_{5}-4.44 x_{6}+\delta_{m} .
\end{aligned}
$$

Fig. 11 shows the four Argand or phasor diagrams [15] (one for each mode of motion) for the eigenvectors of the system of (17). Fig. 12 shows the corresponding force and moment polygons [11] for the $x$-force and pitching moment equations for modes 1 and 2 . These polygons provide an efficient means for determining the contributions made by each open-loop force and moment producing device in the solution of the force and moment equations in each mode of motion. The stability derivative notation of [11] has been used in these diagrams. As Fig. 11 indicates, the motion is coupled, i.e., each mode is characterized by contributions from $\theta$ and $x$.

We now wish to show that, for the purposes of determining a set of weighting coefficients for the OCM index of performance, this manual control problem can be partitioned into two approximate state-uncoupled systems. First, the phasor diagrams for each of the modes 3 and 4 in
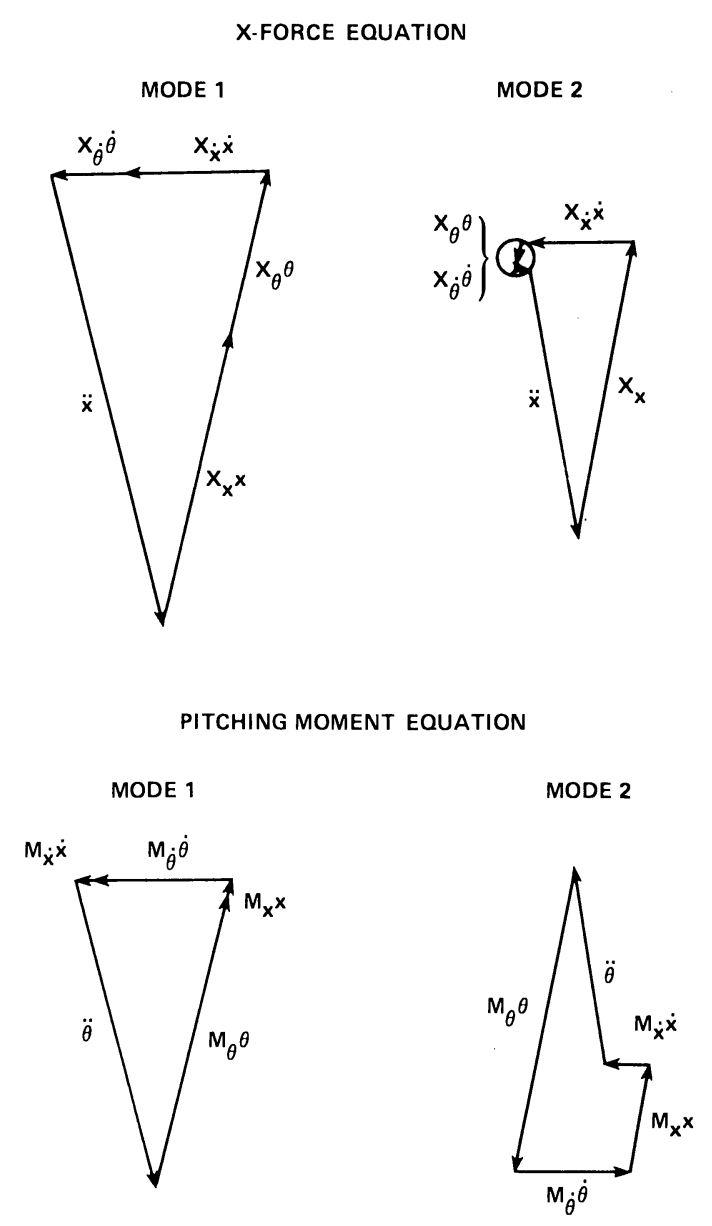

Fig. 12. Force and moment polygons for system of Fig. 10.

Fig. 11 specify initial conditions for (17) which will result in motion involving only the variables shown in the respective diagrams. Here, $f_{1}$ and $\dot{x}$ dominate the initial conditions (and resulting motion) for mode 3 while $f_{2}$ and $\dot{\boldsymbol{\theta}}$ dominate the initial conditions and resulting motion for mode 4 . Thus, the forces $f_{1}$ and $f_{2}$ are, respectively, translation and rotation effectors, since the natural modes in which these variables appear are described almost entirely by either pure translation or rotation. Next, the $x$-force equation polygons for the open-loop modes 1 and 2 indicate that force contributions due to $\theta$ are relatively small. Likewise, the pitching moment equation polygons for the open-loop modes 1 and 2 indicate that moment contributions due to $x$ are relatively small. If we consider that, in the closed-loop modes, $f_{2}$ inputs would be minimizing $\theta$ motion, then a suitable approximation to the $x$-force dynamics can be written as

$$
\begin{aligned}
& \dot{\alpha}_{1}=-10 \alpha_{1}+w_{1} \\
& \dot{\alpha}_{2}=\alpha_{3} \\
& \dot{\alpha}_{3}=-0.00057 \alpha_{1}-60 \alpha_{2}-3 \alpha_{3}+f_{1}+\delta_{f} .
\end{aligned}
$$

Here, $\alpha_{1}$ is a stochastic representation of $f_{2}, \alpha_{2}=x, \alpha_{3}=\dot{x}$ and $w_{1}$ is a white noise input to the shaping filter defining $\alpha_{1}$. Here, we have included the disturbing, control-coupling effects of $f_{2}$ inputs in stochastic fashion in (18) by modeling 
TABLE I

OCM PARAMETERS FOR TASK OF FIG. 10

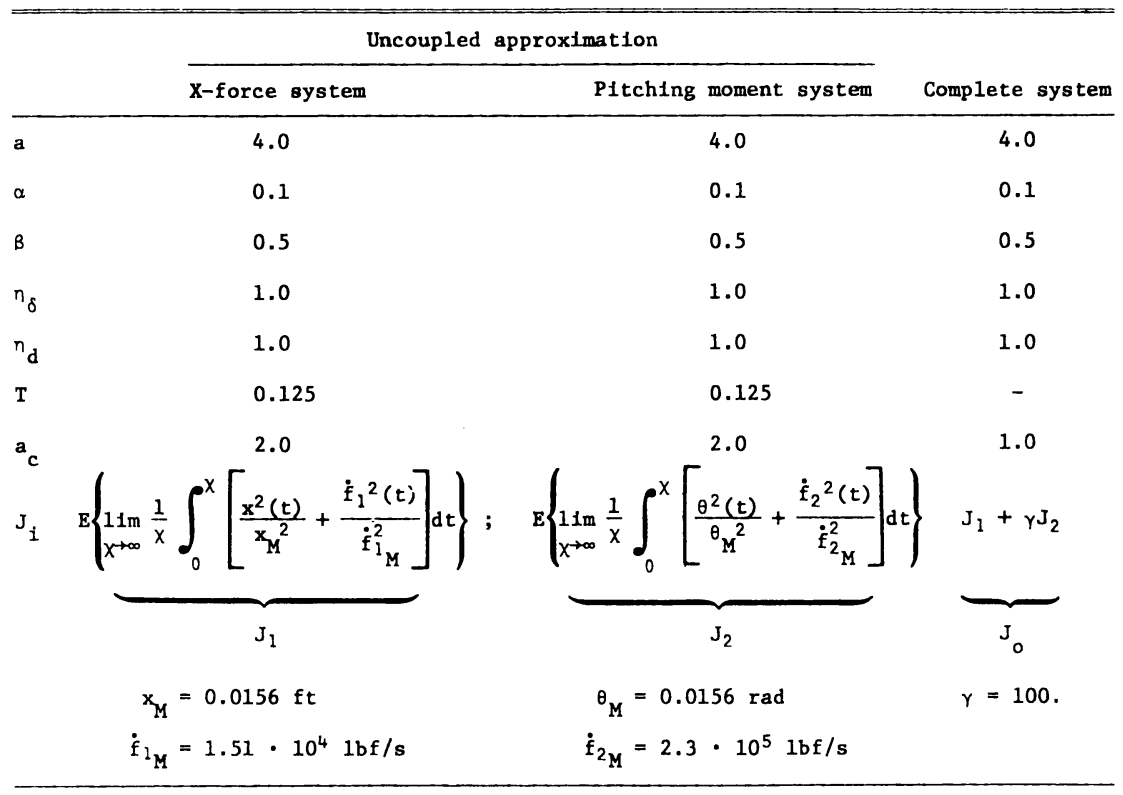

the $f_{2}$ input as the output of a first-order shaping filter excited by white noise.

In a similar manner, $f_{1}$ inputs would be minimizing the $x$ motions in the closed-loop modes, so a suitable approximation to the pitching moment dynamics can be written

$$
\begin{aligned}
& \dot{\beta}_{1}=-10 \beta_{1}+w_{2} \\
& \dot{\beta}_{2}=\beta_{3} \\
& \dot{\beta}_{3}=-0.0004 \beta_{1}-88.8 \beta_{2}-4.44 \beta_{3}+f_{2}+\delta_{m} .
\end{aligned}
$$

Here, $\beta_{1}$ is a stochastic representation of $f_{1}, \beta_{2}=\theta, \beta_{3}=\dot{\theta}$, and $w_{2}$ is a white noise input to the shaping filter defining $\beta_{1}$. As in (18), the disturbing (control-coupling) effects of $f_{1}$ inputs are included in stochastic fashion by modeling $f_{1}$ as the output of a first-order shaping filter excited by white noise. Thus, we have made some approximate but very useful inferences about closed-loop system characteristics from the open-loop eigenanalysis.

An optimal control model of the human operator can now be designed for each of the uncoupled systems of (18) and (19) using the effective time-constant technique previously outlined. Only an estimate of the covariances of $w_{1}$ and $w_{2}$ need be supplied, along with a specification of the spectral characteristics of the disturbances $\delta_{f}$ and $\delta_{m}$. In this latter case, an additional state equation will be added to each of (18) and (19) to describe the disturbance filters. These filters were chosen with a bandwidth of $0.25 \mathrm{rad} / \mathrm{s}$ and root-mean-square (rms) intensities $\sigma_{\delta_{f}}=1000 \mathrm{lbf}, \sigma_{\delta_{m}}$ $=1000 \mathrm{ft}-\mathrm{lbf}$. The covariances of $w_{1}$ and $w_{2}$ were chosen so that $\sigma_{\alpha_{1}}=\sigma_{\delta_{m}}$, and $\sigma_{\beta_{1}}=\sigma_{\delta_{f}}$. Table I lists the pertinent model parameters.

Fig. 13 shows the open-loop transfer functions (product of human operator transfer function and beam system transfer function) which resulted when the OCM effective time-constant method was applied to each of the uncou-
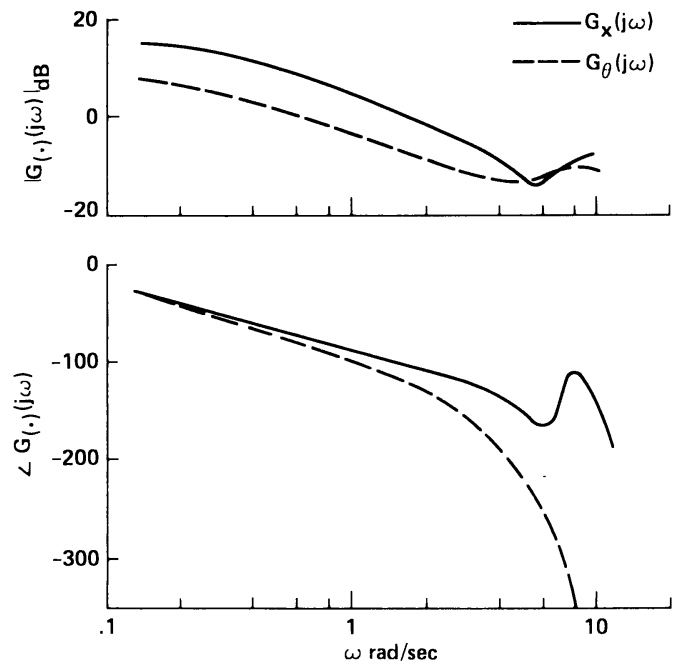

Fig. 13. Human operator-beam open-loop dynamics, operator-transfer function from uncoupled system.

pled systems described by (18) and (19). It should be noted that, although the uncoupled systems were used to obtain the operator transfer function, the complete coupled system (17) was used in calculating the beam transfer functions $x(s) / f_{1}(s)$ and $\theta(s) / f_{2}(s)$.

Just as in Fig. 9, Fig. 13 shows that the human operator and beam combination exhibits $(K / s) e^{-\tau_{e} s}$ characteristics. Again, these characteristics are a necessary condition for the validity of any single-axis tracking model of the human operator. As mentioned previously, one of the purposes of the approximation technique was to allow selection of the OCM index of performance weighting coefficients for the complete coupled task. We write the index of performance for the complete task as a composite of the indices for the 
uncoupled tasks as

$$
J_{0}=J_{1}+\gamma J_{2}
$$

where $J_{1}$ and $J_{2}$ are given in Table I. The parameter $\gamma$ is a factor to be used as a relative weighting adjustment between the two indices $J_{1}$ and $J_{2}$. This weighting is necessary since $x_{M}$ and $\dot{f}_{1_{M}}$ in $J_{1}$ and $\theta_{M}$ and $\dot{f}_{2_{M}}$ in $J_{2}$ were chosen through relations like those of (3) where arbitrary values of $\dot{x}_{M}$ and $\dot{\theta}_{M}$ are selected and used to calculate $x_{M}$ and $\theta_{M}$. The selection of $\gamma$ is accomplished by finding the value that minimizes $\left[\Sigma\left(\partial J / \partial a_{i}\right)_{p}^{2}\right]^{1 / 2}$, where $a_{i}$ is the fraction of attention devoted to the $i$ th observed variable, and the subscript $p$ denotes the projection of the gradient $\partial J / \partial a_{i}$ onto the plane defined by $\sum a_{i}=a_{c}$. Simply stated, this procedure selects the value of $\gamma$ that makes uniform attention allocation to the observed variables the optimum strategy. Here, $\gamma \doteq 100$ minimized the projected gradient. Finally, the attention parameter $a_{c}$ was selected in exactly the same manner as for the single-axis tasks, with a value of $a_{c}=1.0$ meeting the criteria of (8) and (9). Note that no assumptions regarding uncoupled motion are involved in selection $\gamma$ and $a_{c}$.

Comparing the average power in the OCM control forces ( $f_{1}$ and $f_{2}$ ) associated with the four observed variables $x, \dot{x}$, $\theta, \dot{\theta}$ for the system of (17) reveals that the average power in $f_{1}$ due to the model utilizing $\theta$ and $\dot{\theta}$ (a control crosscoupling) is $12 \mathrm{~dB}$ below the average power in $f_{1}$ due to the model utilizing $x$ and $\dot{x}$; conversely, the average power in $f_{2}$ due to the model utilizing $x$ and $\dot{x}$ (a control cross-coupling) is $13 \mathrm{~dB}$ below the average power in $f_{2}$ due to the model utilizing $\theta$ and $\dot{\theta}$. This indicates self-consistency in that the coupled formulation reflects the control-output relations derived from the phasor diagrams for modes 3 and 4 in Fig. 11. Finally, Fig. 14 shows the circulatory transfer functions for the complete problem.

The technique just discussed will next be employed in the design of a flight director for a multiaxis, multioutput system, that is, a light utility helicopter engaged in an instrument flight rules (IFR) landing approach task.

\section{A. A Helicopter Control-Display Design Problem}

A flight director system is one in which the various displayed and/or sensed variables used by the pilot in performing a given task are combined into one instrument, forming a single-axis compensatory tracking task for each control available to the pilot. Perhaps the first device which could be called a flight director was the Sperry Zero Reader [16], developed over 30 years ago. In the intervening years it has been demonstrated that a flight director and the laws that govern the movement of the display elements that constitute the director can significantly reduce the pilot's workload. Indeed, in certain demanding tasks such as the approach and landing of a vertical/short takeoff and landing (V/STOL) aircraft, a well-designed director can be a necessity.

Obviously, flight-director design is not a new topic, and design techniques using analytical pilot models have been
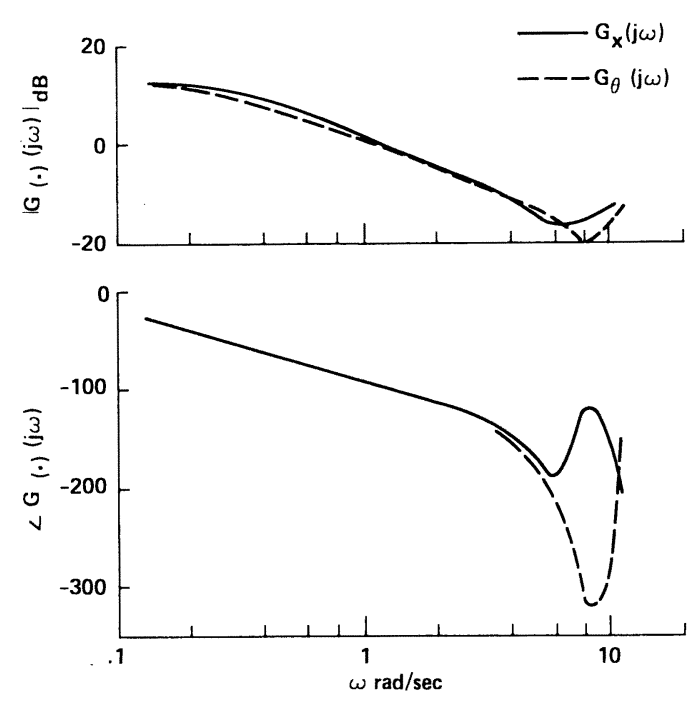

Fig. 14. Human operator-beam open-loop dynamics, operator-transfer function from complete system.

in existence for a number of years [17]-[20]. Each of the existing techniques possesses some limitation, however. The classical designs [17] tend to be somewhat artful and the modern approaches [18]-[20] often do not meet empirically derived pilot-centered requirements.

It is pertinent at this point to summarize the requirements central to the design of acceptable flight-director systems. These can be grouped as 1) guidance and control requirements, and 2) pilot-centered requirements [17]. The first set simply requires adequate closed-loop performance when the pilot uses the director. Adequate performance implies acceptable command following, disturbance regulation, stability, and damping. Of course, these requirements are fundamental to the closed-loop flight-control problem and would hold whether the controller is automatic or human. The second set relates to the fact that the controller is human and for this discussion can be condensed as follows: 1) director-vehicle $K / s$-like characteristics over as large a frequency range as possible, 2) minimum coupling between the directors for each control, and 3) frequency separation of controls. This last requirement arises from the importance of reducing display scanning between directors associated with a single major mode of motion and is usually interpreted as allowing no more than one primary control for each major mode, e.g., one primary control for longitudinal motion and one primary control for lateral motion. It is these last three requirements which often are not met by application of the OCM to director design.

The beam control problem discussed in the preceding section serves as a useful example of a technique which can lead to a flight-director design that meets both sets of requirements just outlined. Indeed, a set of laws which could serve as the basis of a "director" for that task has already been formulated although they have not been referred to as such. Considering the uncoupled system, the operator transfer functions generated by the OCM for the 
beam example can be approximated as:

$$
\begin{aligned}
& \frac{\delta_{f_{1}}}{x}(s) \doteq-\frac{3.5 \cdot 10^{4}}{(s / 0.32+1)} \mathrm{lbf} / \mathrm{ft} \\
& \frac{\delta_{f_{2}}}{\theta}(s) \doteq-\frac{2.5 \cdot 10^{5}}{(s / 0.32+1)} \mathrm{lbf} / \mathrm{rad}
\end{aligned}
$$

where the operator time delays have been omitted. Now consider driving two display indicators by the signals $d_{1}$ and $d_{2}$ where

$$
d_{1}=\frac{\delta_{f_{1}}}{x}(s) \cdot x(s)
$$

and

$$
d_{2}=\frac{\delta_{f_{2}}}{\theta}(s) \cdot \theta(s) .
$$

These signals would constitute a candidate director system for the beam-leveling task in that all of the requirements just enumerated appear to be satisfied. First, acceptable guidance and control characteristics are ensured since those in (21) are the result of a judicious application of an optimal control design technique, which explicitly or implicitly addresses criteria such as disturbance suppression and adequate stability and damping. Second, acceptable operator-centered characteristics are suggested by inspection. For example, the desirable $K / s$-like, open-loop characteristics are seen to obtain in Fig. 13. Minimum coupling between directors is analytically predicted by the low crosscoupling power discussed previously. Frequency separation of control is probably not a factor here since only two director signals are involved in the entire system and display scanning workload would not be a problem.

We will now consider a final example which demonstrates the utility of the technique just developed: the design of a flight-director system for use in a UH- $1 \mathrm{H}$ helicopter on a $-6^{\circ}$ glide slope, constant-speed landing approach. Both longitudinal and lateral motion will be considered. Only the highlights of the design procedure will be discussed since the details of the technique have already been described in detail in this and previous sections. In the following material, we will consider the longitudinal and lateral degrees of freedom of the helicopter to be uncoupled. The entire coupled longitudinal and lateral system is, of course, amenable to the partitioning scheme just outlined; however, for the sake of convenient tutorial presentation, we will assume these two major modes to be uncoupled.

The piloting task consists of minimizing glide slope and localizer deviations (path errors) while maintaining a 60knot ground speed in the presence of vertical and horizontal turbulence. It is assumed that the following variables are displayed to the pilot: groundspeed error, pitch and roll attitude, and glide slope and localizer deviations. The vehicle under consideration has no artificial augmentation but is assumed to possess automatic turn coordination for the lateral mode. Thus, three controls are available to the pilot: longitudinal cyclic pitch $\delta_{E}$ (fore and aft movement of the center stick); collective pitch, $\delta_{C}$ (vertical movement of the collective stick); and lateral cyclic pitch, $\delta_{A}$ (left and right movement of the center stick).

\section{B. Longitudinal System}

Using the preceding task description as a guide, the following longitudinal index of performance can be written:

$$
\begin{aligned}
J_{0}= & E\left\{\operatorname { l i m } _ { \chi \rightarrow \infty } \frac { 1 } { \chi } \int _ { 0 } ^ { \chi } \left[q^{2}(t) / q_{M}^{2}+u^{2}(t) / u_{M}^{2}\right.\right. \\
& +\dot{u}^{2}(t) / \dot{u}_{M}^{2}+h^{2}(t) / h_{M}^{2}+\dot{h}^{2}(t) / \dot{h}_{M}^{2} \\
& \left.\left.+\dot{\delta}_{E}^{2}(t) / \dot{\delta}_{E_{M}}^{2}+\dot{\delta}_{C}^{2}(t) / \dot{\delta}_{C_{M}}^{2}\right] d t\right\} .
\end{aligned}
$$

For reasons similar to those given below (13), attitude variables do not appear in (23). The longitudinal state equations for the UH-1H helicopter at the specified flight condition are

$$
\begin{aligned}
\dot{u}= & -0.028 u+0.835 w-14.14 q-32.1 \theta+23 \alpha \\
& +0.746 \delta_{E}+0.795 \delta_{C} \\
\dot{w}= & 0.011 u-0.8 w+100 q-2.57 \theta+64 \alpha \\
& +2.07 \delta_{E}-9.42 \delta_{C} \\
\dot{q}= & 0.00289 u-0.000129 w-0.753 q-4.33 \alpha \\
& -0.141 \delta_{E}+0.00395 \delta_{C} \\
\dot{\theta}= & q \\
\dot{\alpha}= & 0.16 q-0.303 \alpha \\
\dot{h}= & -w+101.34 \theta
\end{aligned}
$$

where

$u \quad$ groundspeed deviation from desired value ( $\mathrm{ft} / \mathrm{s}$ )

$w$ perturbation velocity component along vehicle $z$ axis $(\mathrm{ft} / \mathrm{s})$;

$q \quad \dot{\theta}(\mathrm{rad} / \mathrm{s})$

$\theta$ pitch attitude deviation from trim (rad);

$\alpha \quad$ state variable associated with stabilizer bar; the stabilizer bar is a mechanical device mounted above the main rotor that acts much like a lagged pitchrate and roll-rate damper for the helicopter;

$h \quad$ glide-slope deviation ( $\mathrm{ft}$ );

$\delta_{E} \quad$ longitudinal cyclic pitch control motion from trim position measured at pilot's hand (in);

$\delta_{C} \quad$ collective pitch control motion from trim position measured at pilot's hand (in).

The observed variables consist of $\theta, q, u, \dot{u}, h$, and $\dot{h}$.

Fig. 15 shows the phasor diagrams for the five longitudinal modes of motion, including the two artificial modes associated with longitudinal cyclic control $\delta_{E}$ and collective control $\delta_{C}$. The force, moment, and kinematic polygons are shown in Figs. 16-18. The phasor diagrams for the control inputs $\delta_{E}$ and $\delta_{C}$ clearly indicate that $\delta_{E}$ is a pitch motion effector and $\delta_{C}$ is a vertical translation effector. The force 
MODE 1

EIGENVALUE:

$-0.512 \pm 0.795 j$

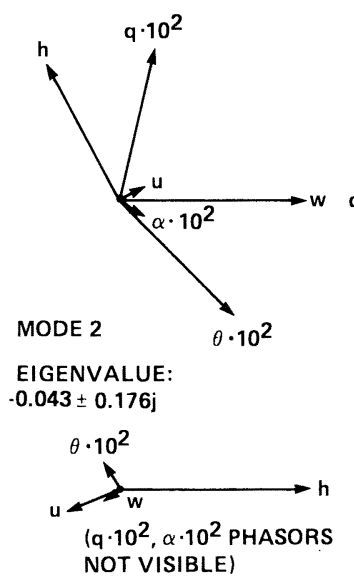

MODE 3

EIGENVALUE:

$-0.775$

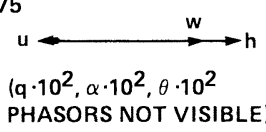

Fig. 15. Eigenvectors for helicopter longitudinal motion.

$$
\begin{aligned}
& \text { X- FORCE EQUATION } \\
& \text { MODE } 1
\end{aligned}
$$
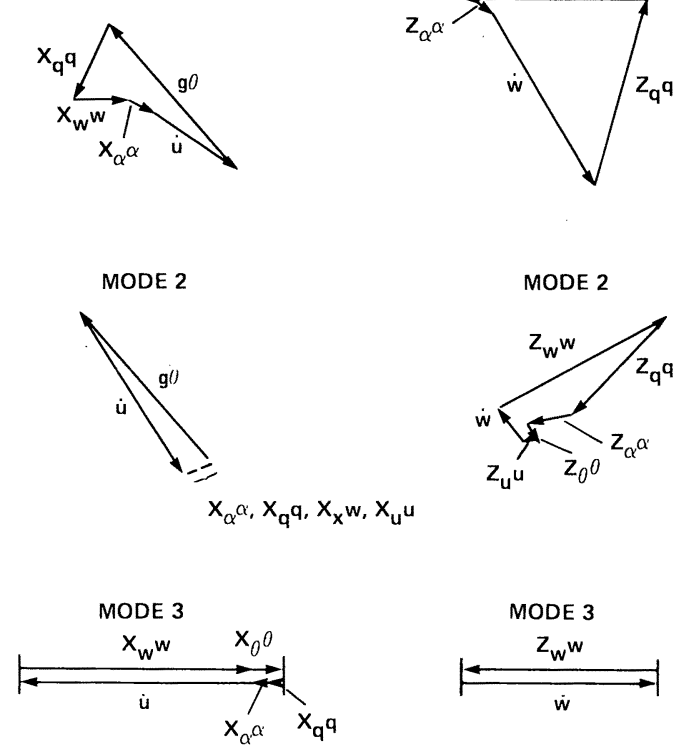

Fig. 16. Force polygons for helicopter longitudinal motion.

and moment polygons suggest the following partitioning of the state equations and variables.

\begin{tabular}{c|l}
\hline Equation (24) & \multicolumn{1}{|c}{ Variables } \\
\hline (a), (c), & $u, q, \theta, \alpha$, with $\delta_{E}$ as control and $\delta_{C}$ as \\
(d), (e); & stochastic disturbance \\
(b), (f) & $\begin{array}{l}w, h, \text { with } \delta_{C} \text { as control and } \delta_{E} \text { as } \\
\text { stochastic disturbance. }\end{array}$ \\
\hline
\end{tabular}

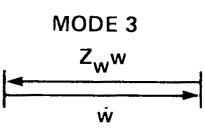

PITCHING MOMENT

EQUATION

STABILIZER BAR

EQUATION

MODE 4

EIGENVALUE:

$-10.0$

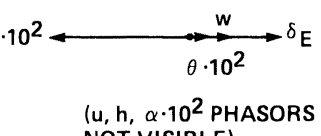

MODE 5

EIGENVALUE:

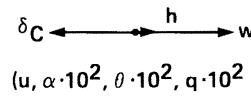

(u, $\alpha \cdot 10^{2}, 0 \cdot 10^{2}, q \cdot 10^{2}$
PHASORS NOT VISIBLE

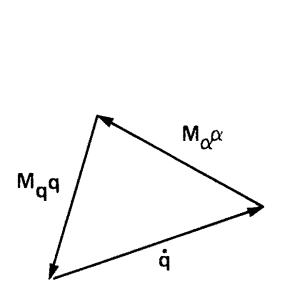

MODE 1

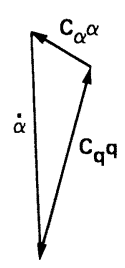

MODE 2
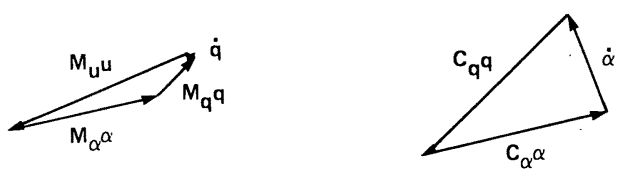

MODE 3
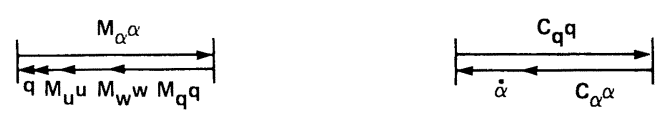

Fig. 17. Pitching moment and stabilizer bar polygons for helicopter longitudinal motion.

KINEMATIC EQUATION

MODE 1

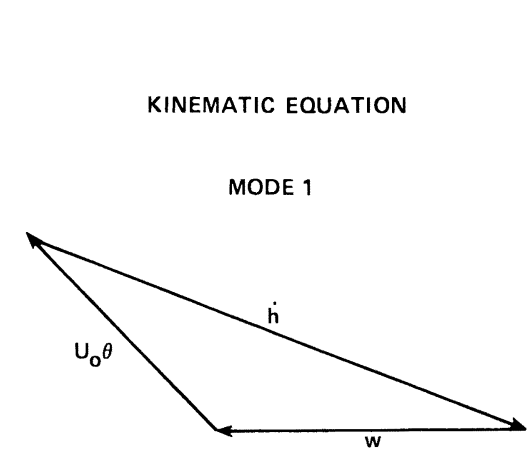

MODE 2

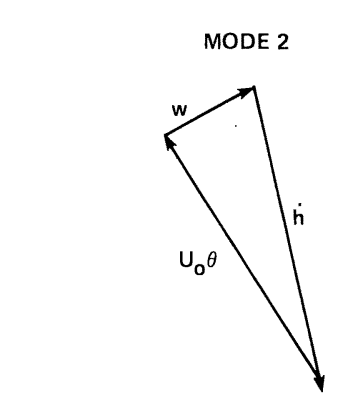

MODE 3

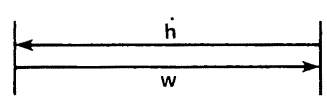

Fig. 18. Kinematic equation polygons for helicopter longitudinal 

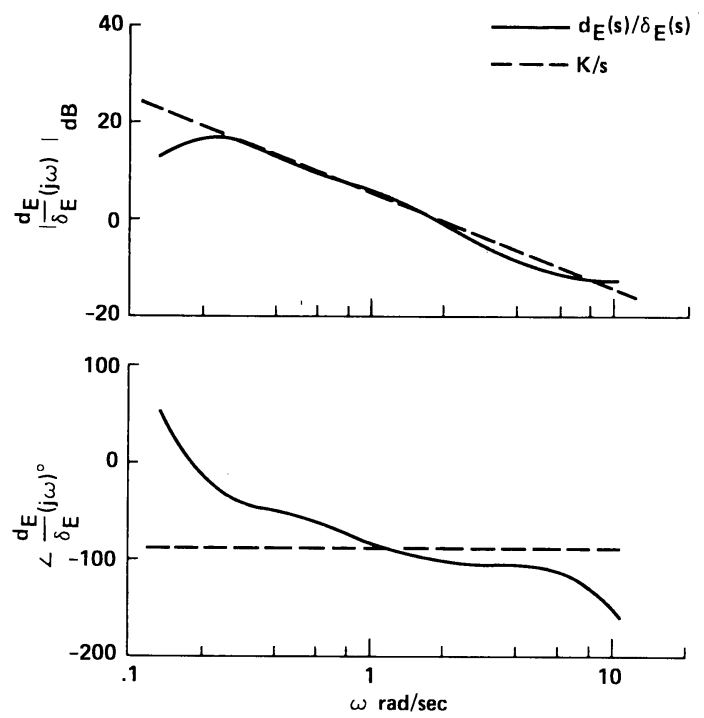

Fig. 19. Flight-director-vehicle dynamics for longitudinal cyclic control.

With this partitioning, indices of performance for the uncoupled systems can be written

$$
\begin{gathered}
J_{1}=E\left\{\operatorname { l i m } _ { x \rightarrow \infty } \frac { 1 } { \chi } \int _ { 0 } ^ { \chi } \left[q^{2}(t) / q_{M}^{2}+\dot{u}^{2}(t) / \dot{u}_{M}^{2}+u^{2}(t) / u_{M}^{2}\right.\right. \\
\left.\left.+\dot{\delta}_{E}^{2}(t) / \dot{\delta}_{E_{M}}^{2}\right] d t\right\} \\
J_{2}=E\left\{\operatorname { l i m } _ { x \rightarrow \infty } \frac { 1 } { \chi } \int _ { 0 } ^ { \chi } \left[h^{2}(t) / h_{M}^{2}+\dot{h}^{2}(t) / \dot{h}_{M}^{2}\right.\right. \\
\left.\left.+\dot{\delta}_{C}^{2}(t) / \dot{\delta}_{C_{M}}^{2}\right] d t\right\} .
\end{gathered}
$$

Just as in the example of the hovering helicopter discussed earlier, the index of performance $J_{1}$ implies $\theta$ and $u$ are, respectively, inner- and outer-loop variables. Since the phasor diagram of Fig. 15 indicates a strong relationship between $\delta_{E}$ and $q$, the selection of $\theta$ as an inner-loop variable and $u$ as an outer-loop variable is indicated.

The equation and variable partitioning just completed along with the definition of $J_{1}$ and $J_{2}$ allow us now to apply the OCM technique outlined in the previous sections. Only the results of this study will be presented. First, equations analogous to (22) provide prospective flight-director laws for the longitudinal cyclic and collective controls. Letting $d_{E}$ and $d_{C}$ represent flight-director command signals for the cyclic and collective controls, respectively, we have

$$
\begin{aligned}
& d_{E}=\frac{\delta_{E}}{\theta}(s) \cdot \theta(s)+\frac{\delta_{E}}{u}(s) \cdot u(s) \\
& d_{C}=\frac{\delta_{C}}{h}(s) \cdot h(s) .
\end{aligned}
$$

Here, $\delta_{E}(s) / \theta(s), \delta_{E}(s) / u(s)$, and $\delta_{C}(s) / h(s)$ are OCMgenerated predictions of pilot transfer functions for the uncoupled equations with time delays omitted. The OCM
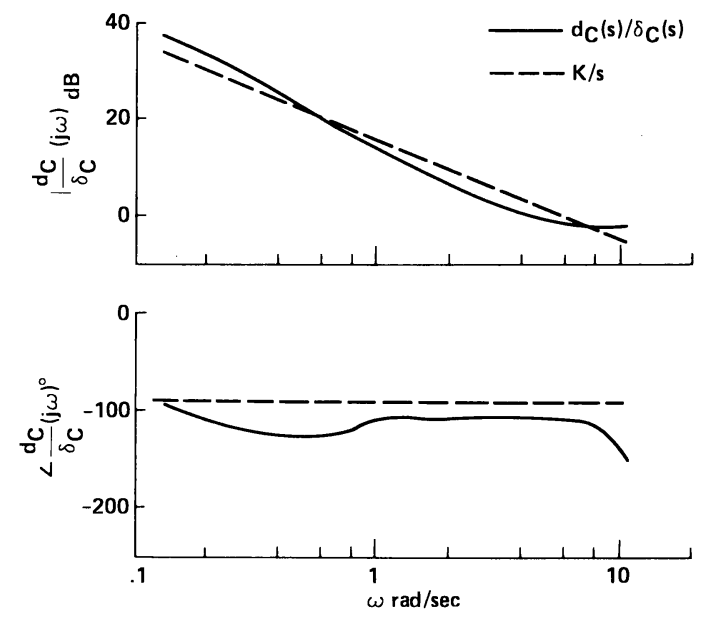

Fig. 20. Flight-director-vehicle dynamics for collective control.

results indicate the following approximations are valid:

$$
\begin{aligned}
& \frac{\delta_{E}}{\theta}(s) \doteq \frac{15.8(s+1)}{(s / 15+1)^{2}} \mathrm{in} / \mathrm{rad} \\
& \frac{\delta_{E}}{u}(s) \doteq-\frac{0.0158(s+1)}{(s / 15+1)^{2}} \mathrm{in} / \mathrm{ft} / \mathrm{s} \\
& \frac{\delta_{C}}{h}(s) \doteq \frac{0.63(s / 1.5+1)}{(s / 15+1)^{2}} \mathrm{in} / \mathrm{ft} .
\end{aligned}
$$

Figs. 19 and 20 show the combined flight-director-vehicle dynamics obtained using (26) and (27a)-(27c). The pertinent vehicle transfer functions for these figures were obtained from the entire set of longitudinal equations, not just the uncoupled approximations. The low-frequency characteristics of the $d_{E}(s) / \delta_{E}(s)$ director-vehicle combination are directly attributable to the poor low-frequency cyclic to pitch attitude dynamic characteristics of the helicopter, itself, not the director design technique. Improving these characteristics requires the design of an appropriate stability augmentation system.

With the exception just noted, it appears that (26) and $(27 \mathrm{a}-\mathrm{c})$ offer an acceptable candidate for a longitudinal flight-director system for this vehicle and task. It would be an informative exercise to use the OCM to evaluate the complete pilot-vehicle system with and without the flightdirector. This was done in a manner completely analogous to the application of the OCM to the beam-leveling control problem. In evaluating the vehicle with director, the observed variables were limited to $d_{E}, \dot{d}_{E}, d_{C}$, and $\dot{d}_{C}$. In reality, of course, pilots would utilize other displayed information $(\theta, h, u$, etc.) in controlling the vehicle. However, it was felt that the pilot should be able to use the directors alone to achieve performance comparable to, if not superior to, that achieved using raw data. Table II summarizes the pertinent model performance predictions, with and without the flight director. Predicted performance with the directors alone was equal to or better than performance with raw data in six of the nine measures; this was accom- 
TABLE II

OCM PERFORMANCE COMPARISONS FOR LONGITUDINAL HELICOPTER CONTROL TASK

\begin{tabular}{cc}
\hline Variable & Relative performance \\
\hline$\sigma_{\mathrm{u}}$ & 0.56 \\
$\sigma_{\mathrm{w}}$ & 0.98 \\
$\sigma_{\mathrm{q}}$ & 0.78 \\
$\sigma_{\theta}$ & 0.42 \\
$\sigma_{\mathrm{h}}$ & 1.35 \\
$\sigma_{\delta_{E}}$ & 0.96 \\
${ }_{\delta_{\mathrm{\delta}}}$ & 1.09 \\
${ }_{\delta_{\mathrm{\delta}}}$ & 0.64 \\
${ }_{\delta_{\mathrm{\delta}}}$ & 1.48 \\
$\mathrm{a}_{\mathrm{C}}$ & 0.55 \\
\hline${ }_{\text {Relative performance }}=$ with \\
director/without director.
\end{tabular}

plished with an attention parameter $a_{c}$ only 55 percent of that for the raw data case.

Fig. 21 shows the predicted pilot transfer functions and control power spectra when using the directors. Ideally, one would like the transfer functions to exhibit pure-gain characteristics across a large frequency range centered at the crossover frequency $\omega_{c}$. While the $\delta_{C}(s) / d_{C}(s)$ function does so, the low-frequency characteristics of the cyclic flight-director-vehicle combination cause a rise in lowfrequency amplitude for $\delta_{E}(s) / d_{E}(s)$. The average power in $\delta_{E}$ due to the model utilizing $d_{C}$ and $\dot{d}_{C}$ (a control cross-coupling) is $17 \mathrm{~dB}$ below the average power in $\delta_{E}$ due to the model utilizing $d_{E}$ and $\dot{d}_{E}$. Likewise, the average power in $\delta_{C}$ due to the model utilizing $d_{E}$ and $\dot{d}_{E}$ (a control cross-coupling) is $22 \mathrm{~dB}$ below the average power in $\delta_{C}$ due to the model utilizing $d_{C}$ and $\dot{d}_{C}$. Thus, the OCM predicts that the criterion for noninteracting controls has been met. Fig. 21 indicates that, in the regions of crossover, the power spectrum for the collective is some 10 to $15 \mathrm{~dB}$ greater than that for the cyclic. The implications of this fïnal result will be discussed after the lateral director is presented.

\section{Lateral System}

Only the results of the lateral cyclic director design will be discussed here. As mentioned previously, it was assumed that tail rotor collective input $\delta_{P}$ was automatically varied to provide turn coordination in the landing approach. Turn coordination implies

where

$$
\dot{\psi}=\frac{g \phi}{U_{0}}+w
$$

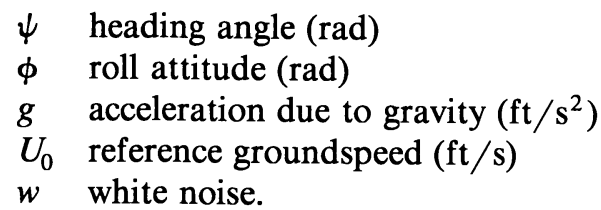

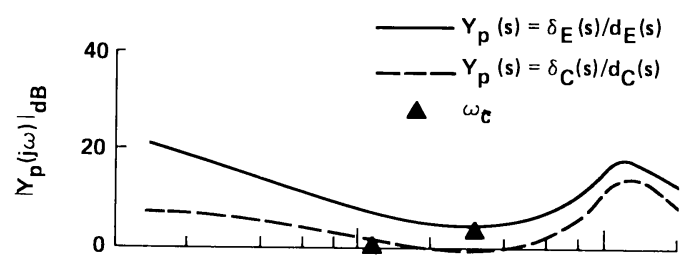

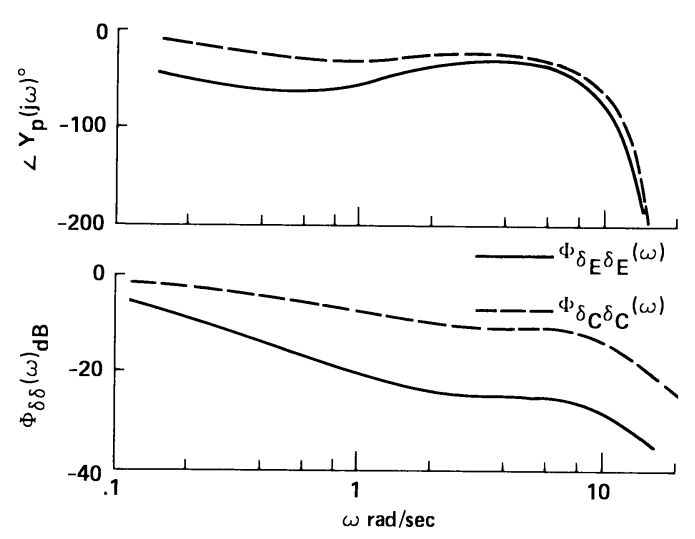

Fig. 21. OCM-transfer functions and power spectra for helicopter with flight directors.

The white noise was added to account for the fact that exact turn coordination is rarely possible. Equation (28) was incorporated into the lateral equations and the tail rotor collective input $\delta_{P}$, which provides the turn coordination, was modeled in stochastic fashion as the output of a first-order filter excited by white noise. The a priori specification that only a single control is available to the pilot to minimize localizer deviations means that the equations of motion need not be approximately uncoupled or partitioned as was the case in the longitudinal design. Innerand outer-loop variable determination is necessary, however; it was shown by an eigenanalysis that $\delta_{A}$ is primarily a roll attitude effector. This meant that roll attitude $\phi$ was an inner-loop variable and lateral deviation $y$ could be considered an outer-loop variable. The following index of performance was utilized:

$$
\begin{aligned}
J_{0}=E\left\{\operatorname { l i m } _ { \chi \rightarrow \infty } \frac { 1 } { \chi } \int _ { 0 } ^ { \chi } \left[\dot{\phi}^{2}(t) / \dot{\phi}_{M}^{2}\right.\right. & +\dot{y}^{2}(t) / \dot{y}_{M}^{2}+y^{2}(t) / y_{M}^{2} \\
& \left.\left.+\dot{\delta}_{A}^{2}(t) / \dot{\delta}_{A_{M}}^{2}\right] d t\right\} .
\end{aligned}
$$

The lateral flight director equation is given by

$$
d_{A}=\frac{\delta_{A}}{\phi}(s) \cdot \phi(s)+\frac{\delta_{A}}{y}(s) \cdot y(s) .
$$

The OCM-generated pilot transfer functions, with time delay deleted, can be approximated as

$$
\begin{aligned}
& \frac{\delta_{A}}{\phi}(s) \doteq \frac{25(s / 1.5+1)^{2}}{(s / 0.15+1)(s / 11+1)^{2}} \text { in } / \mathrm{rad} \\
& \frac{\delta_{A}}{y}(s) \doteq \frac{0.025(s / 1.5+1)}{(s / 11+1)^{2}} \mathrm{in} / \mathrm{ft} .
\end{aligned}
$$

Fig. 22 shows the flight-director-vehicle dynamics for the 

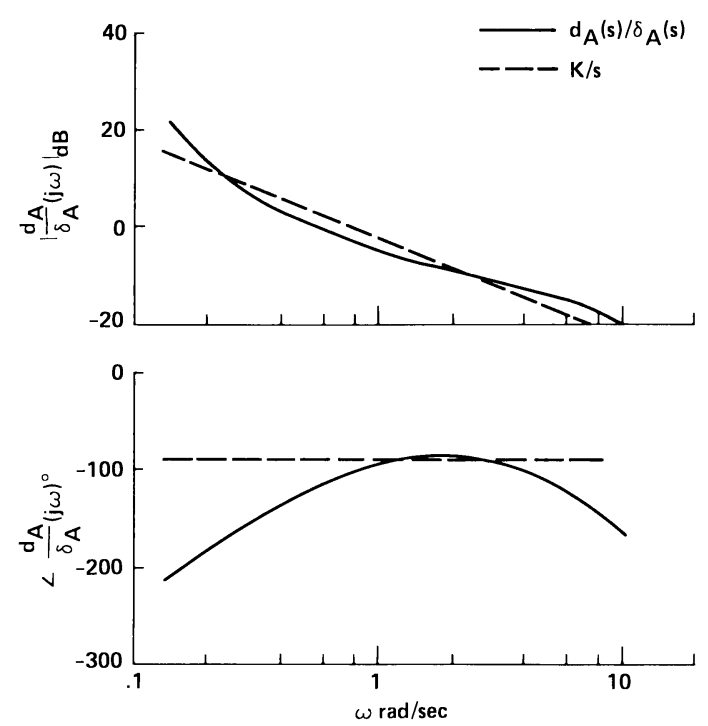

Fig. 22. Flight-director-vehicle dynamics for lateral cyclic control.

lateral case and, as with the longitudinal design, all the requirements for an acceptable director appear to have been met.

\section{Discussion}

The remaining issues of importance in the flight-director design involve frequency separation of control and director display gains. The longitudinal-system modeling results suggest that the collective will be the dominant longitudinal control during the landing approach, while the lateral results show that, by default, the lateral cyclic will be the dominant lateral control. This is less than ideal from the standpoint of scanning workload.

Fig. 23 is a simplified representation of a helicopter electromechanical attitude director indicator (ADI). The dark bars represent the longitudinal and lateral cyclic directors while the circle represents the collective director. In a typical panel installation, the distance between the null position of the cyclic bars and that of the collective subtends about a $4^{\circ}$ angle at the pilot's eye. Since foveal viewing is limited to approximately $2^{\circ}$ subtended visual arc, the pilot must constantly scan between the cyclic and collective null positions in order to track with precision. From the standpoint of scanning workload, a better solution would be to automate the collective control and allow the pilot to concentrate on the cyclic directors. This automation is most easily accomplished by feeding the collective director signal $d_{C}$ to the servo-actuator responsible for collective pitch inputs in the automatic system (assuming such a servo exists).

The issue of flight-director display gains has not been addressed in this discussion of the OCM-modeling technique. Recalling that $K / s$-like "effective vehicle" characteristics occur when equations such as (24) and (30) are implemented, one can write

$$
\frac{d_{i}}{\delta_{i}}(s) \doteq \frac{K_{i}}{s},
$$

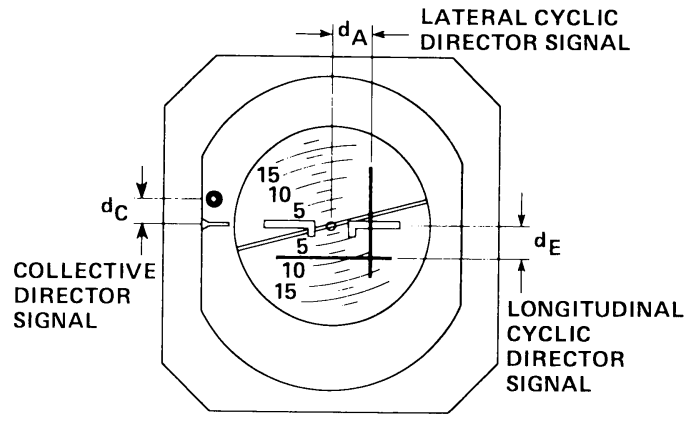

Fig. 23. Typical electromechanical attitude director indicator.

where $K_{i}$ has units of inches per second of display indicator movement per inch of control stick deflection. Based upon pilot-in-the-loop simulation results, [17] suggests $K_{i}$ $\doteq 0.4 \mathrm{in} / \mathrm{s}$-in, while [21] suggests values of 0.08 to 0.1 in/s-in. Since a priori selection of these gains with the OCM technique utilized here does not appear feasible at present, simulator evaluation with initial gain values in the range 0.1 to $0.4 \mathrm{in} / \mathrm{s}$-in appears to be the proper course to take.

\section{Preliminary Evaluation}

Preliminary evaluation of the flight director laws developed here was undertaken in a fixed-base simulator and in actual flight test with the Ames Research Center UH-1H helicopter and V/STOLAND digital avionics system [22]. The simulator included full nonlinear vehicle equations of motion and the same avionics hardware as used in the flight test. Prior to simulator evaluation, the flight-director laws were modified by washing out the low-frequency pitch and roll attitude variables $\theta$ and $\phi$ to avoid possible "standoff" errors in the director logic. A stand-off error occurs when the low-frequency portion of two or more of the variables contributing to a director command effectively cancel each other. In addition, the director laws were simplified by omitting the high-frequency first-order lags in the directors, which occur at $15 \mathrm{rad} / \mathrm{s}$ and $11 \mathrm{rad} / \mathrm{s}((27)$ and (31)). Thus, the modified director laws were given by

$$
\begin{aligned}
& d_{E}=K_{E}\left\{[15.8(s+1)] \theta_{\mathrm{wo}}-0.0158(s+1) u\right\} \\
& d_{C}=K_{C}[0.63(s / 1.5+1) h] \\
& d_{A}=K_{A}\left[\frac{25(s / 1.5+1)^{2}}{(s / 0.15+1)} \phi_{\mathrm{wo}}+0.025(s / 1.5+1) y\right]
\end{aligned}
$$

where $\theta_{\mathrm{wo}}$ and $\phi_{\mathrm{wo}}$ refer to washed-out attitude variables described above. Based on simulation results, the gains on the variables $u, \dot{y}$, and $\dot{h}$ were increased by factors of approximately 4,10 , and 4 , respectively. The $K / s$-like characteristics were not appreciably altered by these gain charges nor by the elimination of the high-frequency phase lags. Acceptable values of $K_{E}, K_{C}$, and $K_{A}$ were selected, 


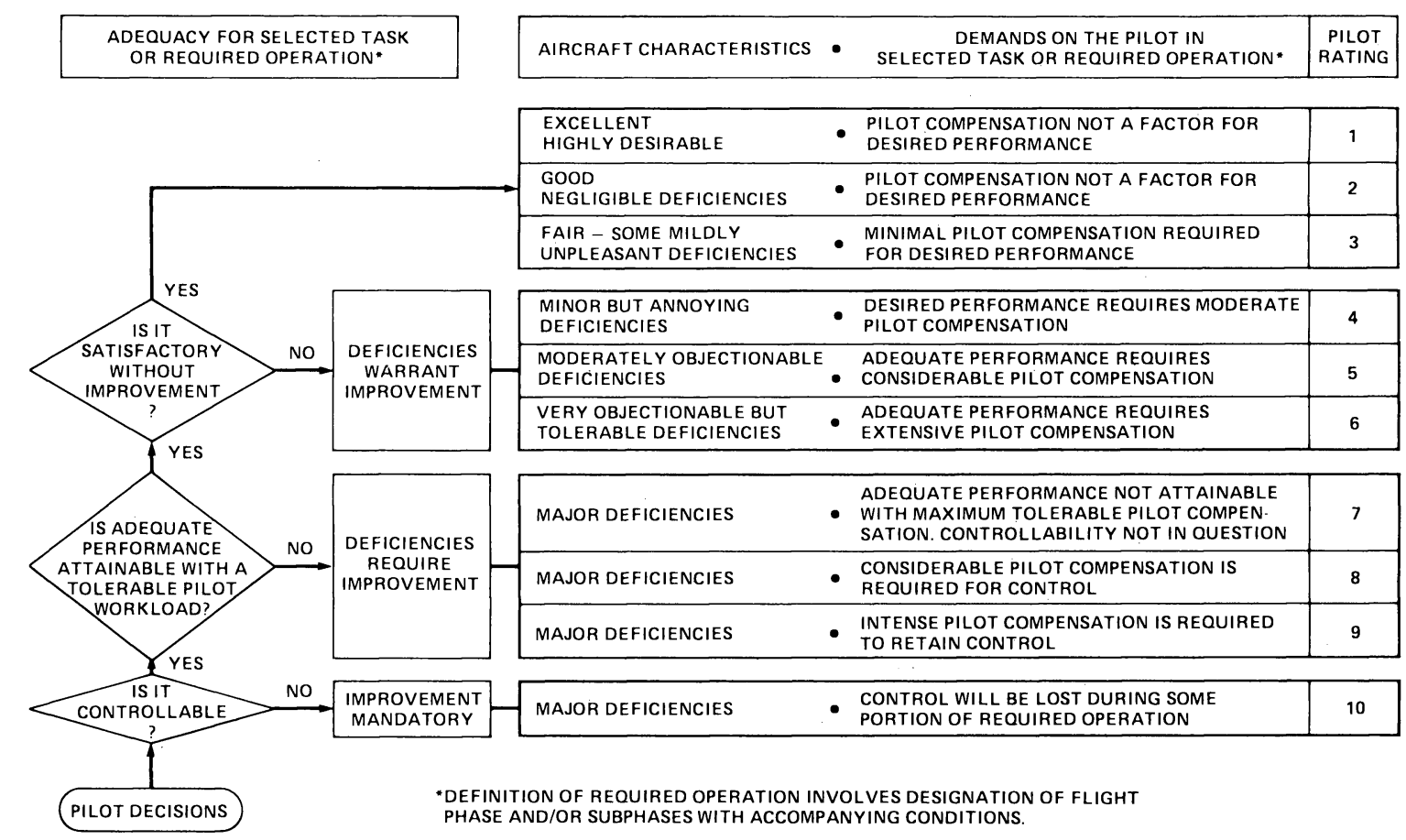

Fig. 24. Cooper-Harper pilot opinion rating scale.

which yielded

$$
\begin{aligned}
& \frac{d_{E}}{\delta_{E}}(s) \doteq \frac{0.2}{s} \\
& \frac{d_{C}}{\delta_{C}}(s) \doteq \frac{0.1}{s} \\
& \frac{d_{A}}{\delta_{A}}(s) \doteq \frac{0.14}{s} .
\end{aligned}
$$

The flight test was conducted under instrument flight rules with the evaluation pilot denied out-the-window information. Although the director was designed for a nominal $-6^{\circ}$ glide slope, actual flight tests were conducted at $-3^{\circ},-6^{\circ}$, and $-9^{\circ}$ approaches. For these three approach angles and a nominal airspeed of 60 knots, the director received an average Cooper-Harper numerical rating of 3.3 on the scale shown in Fig. 24. Overall tracking performance was quite satisfactory, although the pilot noted that the sensitivity of the longitudinal director $d_{E}$ was too large.

The only real surprise in the preliminary evaluation was the necessity for the three gain changes just mentioned. The fact that the original values resulted in low-amplitude oscillations in outer-loop variables is felt to be attributable to the following. 1) The model-based design did not include the attitude washouts. The washouts prevent maintenance of trim pitch and roll attitudes with concomitant deviations in the associated outer-loop variables. 2) The position and rate information in the outer loops were obtained from complementary filters. The derivative relationship between estimated positions and estimated velocities in these filters is not preserved when deviations from nominal are small. Reference [23] reported similar difficulties with filtered positions and velocities, which led to continuous low to moderate amplitude oscillations in vehicle outer-loop variables when a flight director was being utilized. This, of course, does not preclude obtaining an effective flight director, but rather that an analytical design must be modified through simulation to provide adequate performance when such problems occur.

\section{CONCLUding Remarks}

The use of the OCM has been demonstrated as a design tool in the determination and evaluation of flight-director laws for a light utility helicopter. It has been shown that both guidance and control requirements and pilot-centered requirements can be met by application of a novel OCMparameter selection technique.

The flight-director design technique discussed here can also provide a useful methodology for the design of automatic systems which may involve manual backup or optional manual control. The idea is simply to drive the servo-actuators for each control with the corresponding flight-director signal. The utility of such a scheme was demonstrated in [23] and has several desirable features. First, since the flight director has been designed to suit the capabilities of the lowest bandwidth system component (i.e., the human pilot), the possibility of servo ratesaturation, etc., is minimized when the aircraft is under automatic control. Second, the automatic system will "fly" the vehicle in a manner similar to the human pilot. This is an important consideration for the human pilot who must act as a failure detector and performance monitor for the automatic system. Finally, as the example of the previous section demonstrates, considering the flight-director laws as candidate automatic control laws allows the designer to selectively automate certain axes while leaving others subject to manual control. 


\section{REFERENCES}

[1] D. L. Kleinman, S. Baron, and W. H. Levison, "An optimal control model of human response," parts I and II, Automatica, vol. 6, pp. 357-369, May 1970.

[2] S. Baron, "A model for human control and monitoring based on modern control theory," J. Cybern. Inform. Sciences, vol. 1, no. 1, 1976.

[3] R. E. Curry, W. C. Hoffman, and L. R. Young, "Pilot modeling for manned simulation," Air Force Flight Dynamics Lab., AFFDLTR-76-124, vol. 1, 1976.

[4] R. A. Hess, "Analytical display design for flight tasks conducted under instrument meteorological conditions," IEEE Trans. Syst., Man, Cybern., vol. SMC-7, no. 6, pp. 453-462, June 1977.

[5] R. E. Curry, D. L. Kleinman, and W. C. Hoffman, "A design procedure for control display systems," Hum. Factors, vol. 19, no. 5, pp. 421-436, Oct. 1977

[6] S. Baron and W. H. Levison, "Display analysis with the optimal control model of the human operator," Hum. Factors, vol. 19, no. 5, pp. 437-458, Oct. 1977.

[7] G. Johannsen and T. Govindaraj, "Optimal control model predictions of system performance and attention allocation and their experimental validation in a display design study," IEEE Trans. Syst., Man, Cybern., vol. SMC-10, no. 5, pp. 249-261, May 1980.

[8] A. E. Bryson and Y. C. Ho, Applied Optimal Control. New York: Wiley, p. 149, 1975.

[9] R. A. Hess, "Prediction of pilot ratings using an optimal pilot model," Hum. Factors, vol. 19, no. 5, pp. 459-475, 1977.

[10] - "A pilot modeling technique for handling qualities research," J. Cybern. Inform. Sciences, 1980, to appear.

[11] D. T. McRuer, I. L. Ashkenas, and D. Graham, Aircraft Dynamics and Automatic Control. Princeton, NJ: Princeton, 1973.

[12] W. H. Levison, "A model-based technique for predicting pilot opinion ratings for large commercial transports," in Proc. Air Force Flight Dynamics Lab. Flying Qualities Symp., Wright-Patterson AFB, OH, Oct. 1979, pp. 43-77.
[13] D. P. Miller and E. W. Vinje, "Fixed-base flight simulator studies of VTOL aircraft handling qualities in hovering and low-speed flight," Air Force Flight Dynamics Lab., AFFDL-TR-76-152, 1968.

[14] D. T. McRuer and E. S. Krendel, "Mathematical models of human pilot behavior," AGARD-AG-188, Jan. 1974.

[15] B. E. Etkin, Dynamics of Atmospheric Flight. New York: Wiley, 1972, p. 324.

[16] S. Kellogg and C. F. Fragola, "The Sperry zero reader," Aeronaut. Eng. Rev., pp. 22-31, Nov. 1949.

[17] R. H. Hoh, R. H. Klein, and W. A. Johnson, "Development of an integrated configuration management/flight director system for piloted STOL approaches," National Aeronautics and Space Administration CR-2883, 1977

[18] R. T. Chen, J. V. Lebacqz, and E. W. Aiken, "A preliminary look at flight director design philosophies for application to a VTOL landing approach flight experiment," in Proc. Tenth Annu. Conf. Manual Contr., Apr. 1974, pp. 551-573.

[19] W. C. Hoffman, R. E. Curry, D. L. Kleinman, W. M. Hollister, and L. R. Young, "Display/control requirements for VTOL aircraft," National Aeronautics and Space Administration CR-145026, 1975.

[20] R. A. Hess, "Application of a model-based flight director design technique to a longitudinal hover task," J. Aircraft, vol. 14, no. 3, Mar. 1977, pp. 265-271.

[21] A. V. Phatak, L. L. Peach, R. A. Hess, V. L. Ross, G. W. Hall, and R. M. Gerdes, "A piloted simulator investigation of helicopter precision decelerating approaches to hover to determine single-pilot IFR (SPIFR) requirements," AIAA Paper 79-1886, Guidance and Control Conf., Boulder, CO, Aug. 1979.

[22] G. A. Baker, D. N. Jaynes, L. D. Corliss, S. Liden, R. B. Merrick, and D. C. Dugan, "V/STOLAND avionics system flight test data on a UH-1H helicopter," National Aeronautics and Space Administration TM-78591, Feb. 1980.

[23] L. G. Hofmann, R. H. Hoh, W. F. Jewell, G. L. Teper, and P. D. Patel, "Development of automatic and manual flight director landing systems for the XV-15 tilt rotor aircraft in helicopter mode," Systems Technology, Inc., Tech. Rep. No. 1092-1, Jan. 1978. 